\title{
COMPACTNESS AND INDEX OF ORDINARY CENTRAL CONFIGURATIONS FOR THE CURVED $N$-BODY PROBLEM
}

\author{
In memoriam of Florin Diacu \\ Shuqiang Zhu \\ School of Economic and Mathematics, Southwestern University of Finance and \\ Economics, \\ Chengdu 611130, China \\ zhusq@swufe.edu.cn
}

\begin{abstract}
For the curved $n$-body problem, we show that the set of ordinary central configurations is away from most singular configurations in $\mathbb{H}^{3}$, and away from a subset of singular configurations in $\mathbb{S}^{3}$. We also show that each of the $n ! / 2$ geodesic ordinary central configurations for $n$ masses has Morse index $n-2$. Then we get a direct corollary that there are at least $\frac{(3 n-4)(n-1) !}{2}$ ordinary central configurations for given $n$ masses if all ordinary central configurations of these masses are non-degenerate.
\end{abstract}

Key Words: curved $n$-body problem; ordinary central configurations; geodesic configurations; Morse index; compactness; relative equilibrium; hyperbolic relative equilibrium.

\section{INTRODUCTION}

The curved $n$-body problem studies the motion of particles interacting under the cotangent potential in 3-dimensional sphere and 3dimensional hyperbolic sphere. It is a natural extension of the Newtonian $n$-body problem in $\mathbb{R}^{3}$. It roots in the research of Bolyai and Lobachevsky. For history and recent advances, one can refer to Arnold et al. [2], Borisov et al. [3] and Diacu [6]. There are many researches in this area over the past two decades on the Kepler problem, two-body problem, relative equilibria, stability of periodic orbits, etc.

The curved $n$-body problem is a Lagrangian mechanical system. A solution of the Euler-Lagrange's equation in the form of $A(t) \mathbf{q}$ is called a relative equilibrium if $A(t)$ is a 1-parameter subgroup of the symmetry group. The topic of relative equilibria has received much attention recently (see $[4,6,7,8,12,13,20,24,27]$ among others). Unlike that

of the Newtonian $n$-body problem in $\mathbb{R}^{3}$, the set of relative equilibria 
is divided into five classes, see Section 2.3. Diacu, Stoica and Zhu introduce a unified criterion for relative equilibria of the curved $n$-body problem. The configurations of relative equilibrium are characterized as critical points of a single function [9]. The criterion simplifies the job of finding relative equilibria [10]. The configurations are called central configurations and the set central configurations is divided into the ordinary ones and the special ones. A counting problem of ordinary central configurations is also proposed, see Section 2.4.

In the Newtonian $n$-body problem, the celebrated problem of the finiteness of configurations of relative equilibrium is still unsolved for $n>5$ up to now $[1,23,25]$. On the other hand, the collinear case is clear. For any $n$ masses, there are exactly $n ! / 2$ collinear configurations of relative equilibrium (Moulton [16]) and their Morse index is $n-2$ [17]. In general the set of normalized configurations of relative equilibrium is known to be compact (Shub [21]) in the configuration space. Moreover, Palmore [18] proved that there are at least $\frac{(3 n-4)(n-1) !}{2}$ configurations of relative equilibrium for given $n$ masses if all configurations of relative equilibrium of these masses are non-degenerate.

The purpose of this paper is to extend the results mentioned above to the curved $n$-body problem. More precisely, we show that the set of ordinary central configurations is away from most singular configurations in $\mathbb{H}^{3}$, and away from a subset of singular configurations in $\mathbb{S}^{3}$. We show that there are $n ! / 2$ "collinear" ordinary central configurations and their Morse index is $n-2$ in $\mathbb{H}^{3}$, which also holds in $\mathbb{S}^{3}$ provided that some conditions are satisfied. Furthermore, Palmore's estimation also holds.

The paper is organized as follows. In Section 2, we review the $n$ body problem in the three manifolds, $\mathbb{R}^{3}, \mathbb{S}^{3}$ and $\mathbb{H}^{3}$, the criterion for relative equilibria and the counting of ordinary central configurations. Then we state our main results. In Section 3, we prove the results on compactness of the set of ordinary central configurations. In Section 4, we prove the results on the number and Morse index of the geodesic ordinary central configurations. In Appendix, we discuss association between the central configurations and the relative equilibria motions.

\section{Relative EQUilibRIA OF THE CURVED $n$-BODY PROBLEM AND MAIN RESULTS}

In this section, we first briefly review the $n$-body problem on the three manifolds, $\mathbb{R}^{3}, \mathbb{S}^{3}$ and $\mathbb{H}^{3}$, criterion for relative equilibria, then state the main results of this paper. Vectors are all column vectors, but 
written as row vectors in the text. The masses $m_{1}, \ldots, m_{n}$ are always positive.

2.1. Relative equilibria of the $n$-body problem in $\mathbb{R}^{3}$. The Newtonian $n$-body problem in $\mathbb{R}^{3}$ studies the motion of $n$ particles in $R^{3}$ with masses $m_{1}, \ldots, m_{n}$ under the gravitational interaction. It is a Lagrange mechanical system with Lagrangian function

$$
L=\frac{1}{2} \sum_{i=1}^{n} m_{i} \dot{\mathbf{q}}_{i} \cdot \dot{\mathbf{q}}_{i}-U_{0}(\mathbf{q}),
$$

where $\mathbf{q}=\left(\mathbf{q}_{1}, \ldots, \mathbf{q}_{n}\right), \mathbf{q}_{i}=\left(x_{i}, y_{i}, z_{i}\right) \in \mathbb{R}^{3}$, and $U_{0}=\sum \frac{m_{i} m_{j}}{\left\|\mathbf{q}_{i}-\mathbf{q}_{j}\right\|}$ is the potential defined on the configuration space $\left(\mathbb{R}^{3}\right)^{n}-\Delta, \Delta=$ $\cup_{1 \leq i<j \leq n}\left\{\mathbf{q} \in\left(\mathbb{R}^{3}\right)^{n} \mid \mathbf{q}_{i}=\mathbf{q}_{j}\right\}$

$\mathrm{A}$ relative equilibrium is an integral curve of the system in the form of $A(t) \mathbf{q}$, with $A(t)$ being a uniform rotation in $S O(3)$. The corresponding configuration $\mathbf{q}$ is called a configuration of relative equilibrium, Every uniform rotation in $S O(3)$ has an fixed axis. Assume that the $z$-axis is the rotation axis. Then it is well-known that the configurations of relative equilibrium must be on a plane perpendicular to the $z$ axis. Without lose of generality, we assume that they are on the plane $\{z=0\}$, then they are critical points of $U_{0}-\lambda I_{0}$ for some $\lambda \in \mathbb{R}$, where $I_{0}(\mathbf{q})=\sum m_{i}\left(x_{i}^{2}+y_{i}^{2}\right)$.

Two configurations of relative equilibrium are said to be in one class if one can be deduced from the other by some rotation in $S O(2)$ and nonzero scalar multiplication. The finiteness problem on configurations of relative equilibrium is: given $n$ masses $m_{1}, \ldots, m_{n}$, is the number of classes of configurations of relative equilibrium finite? In other words, is the number of configurations of relative equilibrium in $\left\{\mathbf{q} \in\left(\mathbb{R}^{2}\right)^{n}-\right.$ $\left.\Delta I_{0}(\mathbf{q})=1\right\} / S^{1}$ finite? For history and advance of this problem, we refer the readers to $[1,23,25]$ and the references therein.

2.2. The curved $n$-body problem in $\mathbb{S}^{3}$ and $\mathbb{H}^{3}$. The curved $n$ body problem studies the motion of $n$ particles interacting under the so-called cotangent potential in $\mathbb{S}^{3}$ and $\mathbb{H}^{3}$. The two manifolds can be parameterized in many ways. The Cartesian coordinates are convenient in many cases. That is, $\mathbb{S}^{3}$ (resp. $\mathbb{H}^{3}$ ) is the unit sphere in $\mathbb{R}^{4}$ (resp. $\left.\mathbb{R}^{3,1}\right)$. Recall that the'inner product in those 4-dimensional linear space are $\mathbf{q}_{1} \cdot \mathbf{q}_{2}=x_{1} x_{2}+y_{1} y_{2}+z_{1} z_{2}+\sigma w_{1} w_{2}$, where $\mathbf{q}_{i}=\left(x_{i}, y_{i}, z_{i}, w_{i}\right), \sigma=1$ for $\mathbb{R}^{4}$ and $\sigma=-1$ for $\mathbb{R}^{3,1}$. Then $\mathbb{S}^{3}=\left\{(x, y, z, w) \in \mathbb{R}^{4} \mid x^{2}+y^{2}+\right.$ $\left.z^{2}+w^{2}=1\right\}, \mathbb{H}^{3}=\left\{(x, y, z, w) \in \mathbb{R}^{3,1} \mid x^{2}+y^{2}+z^{2}-w^{2}=-1, w \geq 1\right\}$. The Riemannian metrics on $\mathbb{S}^{3}$ and $\mathbb{H}^{3}$ are induced from the "inner product". The distance between two point masses $m_{i}$ and $m_{j}, d_{i j}=$ 
$d\left(\mathbf{q}_{i}, \mathbf{q}_{j}\right)$, is computed by $\cos d_{i j}=\mathbf{q}_{i} \cdot \mathbf{q}_{j}$ on $\mathbb{S}^{3}$ and $\cosh d_{i j}(\mathbf{q})=-\mathbf{q}_{i} \cdot \mathbf{q}_{j}$ on $\mathbb{H}^{3}$.

The curved $n$-body problem in $\mathbb{S}^{3}$ is a Lagrange mechanical system with Lagrangian function

$$
L=\frac{1}{2} \sum_{i=1}^{n} m_{i} \dot{\mathbf{q}}_{i} \cdot \dot{\mathbf{q}}_{i}-U_{1}(\mathbf{q})
$$

where $\mathbf{q}=\left(\mathbf{q}_{1}, \ldots, \mathbf{q}_{n}\right), \mathbf{q}_{i} \in \mathbb{S}^{3}$. Let $\Delta_{+}$be the singular set $\Delta_{+}=$ $\cup_{1 \leq i<j \leq n}\left\{\mathbf{q} \in\left(\mathbb{S}^{3}\right)^{n} \mid \mathbf{q}_{i}= \pm \mathbf{q}_{j}\right\}$. The potential is $U_{1}=\sum m_{i} m_{j} \cot d_{i j}$ defined on the configuration space $\left(\mathbb{S}^{3}\right)^{n}-\Delta_{+}$. The equations of motion are $[6,9]$ :

$$
\left\{\begin{array}{l}
m_{i} \ddot{\mathbf{q}}_{i}=\sum_{j=1, j \neq i}^{n} \frac{m_{i} m_{j}\left[\mathbf{q}_{j}-\cos d_{i j} \mathbf{q}_{i}\right]}{\sin ^{3} d_{i j}}-\sigma m_{i}\left(\dot{\mathbf{q}}_{i} \cdot \dot{\mathbf{q}}_{i}\right) \mathbf{q}_{i} \\
\mathbf{q}_{i} \cdot \mathbf{q}_{i}=\sigma, \quad i=1, \ldots, n .
\end{array}\right.
$$

Recall that $\sigma=1$ for $\mathbb{S}^{3}$ and $\sigma=-1$ for $\mathbb{H}^{3}$.

Likewise, the curved $n$-body problem in $\mathbb{H}^{3}$ is a Lagrange mechanical system with Lagrangian function

$$
L=\frac{1}{2} \sum_{i=1}^{n} m_{i} \dot{\mathbf{q}}_{i} \cdot \dot{\mathbf{q}}_{i}-U_{-1}(\mathbf{q}),
$$

where $\mathbf{q}=\left(\mathbf{q}_{1}, \ldots, \mathbf{q}_{n}\right), \mathbf{q}_{i} \in \mathbb{H}^{3}$. Let $\Delta_{-}$be the singular set $\Delta_{-}=$ $\cup_{1 \leq i<j \leq n}\left\{\mathbf{q} \in\left(\mathbb{H}^{3}\right)^{n} \mathbf{q}_{i}=\mathbf{q}_{j}\right\}$. The potential is $U_{-1}(\mathbf{q})=\sum m_{i} m_{j} \operatorname{coth} d_{i j}$ defined on the configuration space $\left(\mathbb{H}^{3}\right)^{n}-\Delta_{-}$. Replacing the trigonometrical functions by the hyperbolic ones and putting $\sigma=-1$, equations (1) become the equations of motion for the curved $n$-body problem in $\mathbb{H}^{3}$.

2.3. Relative equilibria in $\mathbb{S}^{3}$ and $\mathbb{H}^{3}$. A simple mechanical system with symmetry in the terminology of Smale is a Lagrange mechanical system on a manifold $M$ in the form of $L=K(\dot{\mathbf{q}})+U(\mathbf{q})$, where $K$ is a Riemannian metric on $M$ and there is a Lie group $G$ acting on $M$ preserving $K$ and $U$ smoothly. A solution of the Euler-Lagrange's equation in the form of $A(t) \mathbf{q}$ is called a relative equilibrium if $A(t)$ is a 1-parameter subgroup of the group $G$. It is well-known that the corresponding configuration $\mathbf{q}$ is a critical point of the augmented potential

$$
U_{\xi}(\mathbf{q})=U(\mathbf{q})+K\left(\xi_{M}(\mathbf{q})\right),
$$

where $\xi$ belongs to the Lie algebra of $G$ and $\xi_{M}(\mathbf{q})=\left.\frac{d}{d s}\right|_{s=0} \exp (s \xi) \mathbf{q}$ is the vector field on $M$ generated by $\xi[15,22]$.

The curved $n$-body problem in $\mathbb{S}^{3}$ (resp. $\mathbb{H}^{3}$ ) is a simple mechanical system with symmetry $O(4)$ (resp. $O(3,1)$ ), the set of matrices that keeps the inner product in $\mathbb{R}^{4}$ (resp. $\mathbb{R}^{3,1}$ ). Let $\xi$ be some element in the 
Lie algebra of $O(4)(O(3,1))$. Then the 1-parameter subgroup of $O(4)$ $(O(3,1))$ takes the form of $\exp (t \xi)$, and the corresponding vector field on the configuration space is $\left(\xi \mathbf{q}_{1}, \ldots, \xi \mathbf{q}_{n}\right)$. The augmented potential takes the form of

$$
U_{1}+\frac{1}{2} \sum_{i=1}^{n} m_{i} \xi \mathbf{q}_{i} \cdot \xi \mathbf{q}_{i}, \quad \text { or } \quad U_{-1}+\frac{1}{2} \sum_{i=1}^{n} m_{i} \xi \mathbf{q}_{i} \cdot \xi \mathbf{q}_{i} .
$$

This coordinates-free way is adopted in the study of relative equilibria in the Newtonian $n$-body problem in higher dimensions, see $[5,19]$.

It is convenient to use coordinates for our problem. Note that each 1-parameter subgroup is conjugate to

$$
\begin{aligned}
A_{\alpha, \beta}(t)= & {\left[\begin{array}{cccc}
\cos \alpha t & -\sin \alpha t & 0 & 0 \\
\sin \alpha t & \cos \alpha t & 0 & 0 \\
0 & 0 & \cos \beta t & -\sin \beta t \\
0 & 0 & \sin \beta t & \cos \beta t
\end{array}\right] \text { in } O(4) \text { and }, } \\
B_{\alpha, \beta}(t)= & {\left[\begin{array}{cccc}
\cos \alpha t & -\sin \alpha t & 0 & 0 \\
\sin \alpha t & \cos \alpha t & 0 & 0 \\
0 & 0 & \cosh \beta t & \sinh \beta t \\
0 & 0 & \sinh \beta t & \cosh \beta t
\end{array}\right] \text { in } O(3,1) . }
\end{aligned}
$$

We have neglected the 1-parameter subgroups of $S O(3,1)$ that represent the parabolic rotations since they do not lead to relative equilibria of the curved n-body problem, [6, 7]. Following Diacu, [6], in $\mathbb{S}^{3}$, we call relative equilibria elliptic if only one of $\alpha$ and $\beta$ is nonzero, and elliptic-elliptic if $\alpha \beta \neq 0$; in $\mathbb{H}^{3}$, we call relative equilibria elliptic if $\alpha \neq 0, \beta=0$, hyperbolic if $\alpha=0, \beta \neq 0$, and elliptic-hyperbolic if $\alpha \beta \neq 0$.

The Lie algebra elements corresponding to $A_{\alpha, \beta}(t)$ and $B_{\alpha, \beta}(t)$ are

$$
\left[\begin{array}{cccc}
0 & -\alpha & 0 & 0 \\
\alpha & 0 & 0 & 0 \\
0 & 0 & 0 & -\beta \\
0 & 0 & \beta & 0
\end{array}\right],\left[\begin{array}{cccc}
0 & -\alpha & 0 & 0 \\
\alpha & 0 & 0 & 0 \\
0 & 0 & 0 & \beta \\
0 & 0 & \beta & 0
\end{array}\right]
$$

respectively. Hence, the function $K\left(\xi \mathbf{q}_{1}, \ldots, \xi \mathbf{q}_{n}\right)$ for the $\mathbb{S}^{3}$ case is $\alpha^{2} \sum_{i=1}^{n} m_{i}\left(x_{i}^{2}+y_{i}^{2}\right) / 2+\beta^{2} \sum_{i=1}^{n} m_{i}\left(z_{i}^{2}+w_{i}^{2}\right) / 2$. Note that $\mathbf{q}_{i} \cdot \mathbf{q}_{i}=1$. The function reduces to

$$
\frac{\alpha^{2}-\beta^{2}}{2} \sum_{i=1}^{n} m_{i}\left(x_{i}^{2}+y_{i}^{2}\right)+\frac{\beta^{2}}{2} \sum_{i=1}^{n} m_{i} .
$$


Similarly, the function $K\left(\xi \mathbf{q}_{1}, \ldots, \xi \mathbf{q}_{n}\right)$ for the $\mathbb{H}^{3}$ case reduces to

$$
\frac{\alpha^{2}+\beta^{2}}{2} \sum_{i=1}^{n} m_{i}\left(x_{i}^{2}+y_{i}^{2}\right)+\frac{\beta^{2}}{2} \sum_{i=1}^{n} m_{i} \text {. }
$$

Let $I_{1}(\mathbf{q})=\sum_{i=1}^{n} m_{i}\left(x_{i}^{2}+y_{i}^{2}\right)\left(\operatorname{resp} . I_{-1}(\mathbf{q})=\sum_{i=1}^{n} m_{i}\left(x_{i}^{2}+y_{i}^{2}\right)\right)$ be the momentum of inertia for configurations $\mathbf{q}$ in $\mathbb{S}^{3}$ (resp. $\left.\mathbb{H}^{3}\right)$. Let

$$
S_{c}^{+}=I_{1}^{-1}(c)-\Delta_{+}, \quad S_{c}^{-}=I_{-1}^{-1}(c)-\Delta_{-} .
$$

We have the following criterion of relative equilibria.

Theorem 1 ([9]). For the curved $n$-body problem, $A_{\alpha, \beta}(t) \mathbf{q}\left(B_{\alpha, \beta}(t) \mathbf{q}\right)$ is a relative equilibrium if and only if the configuration $\mathbf{q}$ is a critical point of

$U_{1}(\mathbf{q})+\frac{\alpha^{2}-\beta^{2}}{2} \sum_{i=1}^{n} m_{i}\left(x_{i}^{2}+y_{i}^{2}\right)$, or $U_{-1}(\mathbf{q})+\frac{\alpha^{2}+\beta^{2}}{2} \sum_{i=1}^{n} m_{i}\left(x_{i}^{2}+y_{i}^{2}\right)$.

Thus all relative equilibria, no matter it is elliptic, elliptic-elliptic, hyperbolic, or elliptic-hyperbolic, can be obtained by finding configurations that are critical points of one function

$$
U_{1}-\lambda I_{1} \text {, or }\left.U_{1}\right|_{S_{c}^{+}}, \quad\left(\operatorname{resp} . U_{-1}-\lambda I_{-1} \text {, or }\left.U_{-1}\right|_{S_{c}^{-}}\right) \text {. }
$$

This unity is obtained by restricting the relative equilibria to those in the form of $A_{\alpha, \beta}(t) \mathbf{q}\left(B_{\alpha, \beta}(t) \mathbf{q}\right)$ and by studying relative equilibria in the 3-dimensional space. For example, in the hyperbolic case, if we study the relative equilibria on a 2-dimensional physical space, then the augmented potentials for the elliptic relative equilibria and the hyperbolic ones are different, see $[7,11]$.

Let $\mathbf{q}$ be a critical point of (3). Then it leads to infinitely many relative equilibria $A_{\alpha, \beta}(t) \mathbf{q}$ with $\lambda$ equals $-\frac{\alpha^{2}-\beta^{2}}{2}\left(\operatorname{resp} . B_{\alpha, \beta}(t) \mathbf{q}\right.$ with $\lambda=-\frac{\alpha^{2}+\beta^{2}}{2}$ ), which maybe elliptic or elliptic-elliptic (resp. elliptic, hyperbolic, or elliptic-hyperbolic). So to study the relative equilibria, it is convenient to start with the associated configurations. We will discuss the relationship between the critical points of (3) and relative equilibria in the Appendix.

In $\mathbb{S}^{3}$, the function $U_{1}$ has critical points. Besides relative equilibria, such configurations also lead to equilibria of the system $\mathbf{q}(t)=\mathbf{q}$. Let $\mathbf{q}$ be such a critical point. Then it is a critical point of $U_{1}-\lambda I_{1}$ with $\lambda=0$, or with $\lambda$ being any real value if it is one configuration that lies on $\mathbb{S}_{x y}^{1} \cup S_{z w}^{1}$, two circles to be defined in Section $2.5[6,9,26]$. The property of these configurations and the other critical points of (3) are different in many ways. This motivates the following definition. 
Definition 2 ([9]). A configuration $\mathbf{q}$ is called a central configuration if it is a critical point of (3). If it is a critical point of $U_{1}$, it is a special central configuration or an equilibrium configuration; otherwise, it is an ordinary central configuration. The value $\lambda$ in (3) is the multiplier.

The central configurations defined above are deduced from the relative equilibria, and may not have some nice properties possessed by the well-known central configurations of the Newtonian $n$-body problem. One can refer to [9] for more of their properties. We only discuss the ordinary central configurations in the main part of this paper.

2.4. The counting of ordinary central configurations. Thanks to Theorem 1, we can discuss the counting of ordinary central configurations. Note that the set of ordinary central configurations in $\mathbb{S}^{3}\left(\right.$ resp. $\left.\mathbb{H}^{3}\right)$ is invariant under the action of $S O(2) \times S O(2)$ (resp. $\left.S O(2) \times S O^{+}(1,1)\right)$ on the configuration space. The group $S O^{+}(1,1)$ is $\left\{\left[\begin{array}{cc}\cosh s & \sinh s \\ \sinh s & \cosh s\end{array}\right] \mid s \in \mathbb{R}\right\}$, the identity component of $S O(1,1)$. The symmetry acts on the configuration space in the following way. For instance, in $\mathbb{H}^{3}$, let $\chi=\left(\chi_{1}, \chi_{2}\right) \in S O(2) \times S O^{+}(1,1)$. Then

$$
\chi \mathbf{q}=\left(\chi \mathbf{q}_{1}, \ldots, \chi \mathbf{q}_{n}\right), \chi \mathbf{q}_{i}=\left(\chi_{1}\left(x_{i}, y_{i}\right), \chi_{2}\left(z_{i}, w_{i}\right)\right) .
$$

The quotient of the set of ordinary central configurations under $S O(2) \times$ $S O(2)$ (resp. $S O(2) \times S O^{+}(1,1)$ ) will be called the set of classes of ordinary central configurations.

The major difference between the ordinary central configurations and the configurations of relative equilibrium in the Newtonian case is the lack of homothety symmetry. For the Newtonian $n$-body problem, by the homothety symmetry, the set of configurations of relative equilibrium on $I_{0}^{-1}(c)$ equivalent to that on $I_{0}^{-1}(1)$. So it is enough to do the counting just on $I_{0}^{-1}(1)$. For the curved $n$-body problem, the structure of the set of ordinary central configurations depends on the value of $I_{ \pm 1}(\mathbf{q})$ in an essential way. For instance, for two given masses in $\mathbb{S}^{3}$, the number of ordinary central configurations varies as the value of $I_{1}$ varies [9]. It is also easy to see the existence of critical points of $\left.U_{1}\right|_{S_{c}^{+}}$ and $\left.U_{-1}\right|_{S_{c}^{-}}$for each $c$ in some interval.

Corollary 3 ([9]). For any given $n \geq 2$ masses, there are infinitely many classes of ordinary central configurations in the curved n-body problem in $\mathbb{S}^{3}$ and $\mathbb{H}^{3}$.

Thus, to make the counting problem reasonable, we propose to count ordinary central configurations on $S_{c}^{+}\left(S_{c}^{-}\right)$for different value of $c$. To imitate the finiteness problem on configurations of relative equilibrium 
of the Newtonian $n$-body problem [23], we ask: Are there always only finitely many ordinary central configurations classes on $S_{c}^{+}\left(S_{c}^{-}\right)$for the curved $n$-body problem for almost all choices of masses $\left(m_{1}, \ldots, m_{n}\right)$ ? The answer is negative for some choices of masses. For example, for two masses $m_{1}=m_{2}$ in $\mathbb{S}^{3}$, there are infinitely many classes of ordinary central configurations on $S_{m_{1}}^{+}$, see Section 10 of [9].

2.5. Main results. We are interested in the investigation of the set of the ordinary central configurations. We first consider the compactness, then focus on the counting of geodesic ordinary central configurations and their Morse index. We postpone the proofs of Proposition 4, Theorem 5 and 7 to Section 3, the proofs of Theorem12 and 14 to Section 4.

In $\mathbb{H}^{3}$, similar to the singular set of the Newtonian n-body problem, a point in $\Delta_{-}$can be written as

$$
X=\left(\mathbf{q}_{1}^{\prime}, \ldots, \mathbf{q}_{k_{1}}^{\prime}, \mathbf{q}_{k_{1}+1}^{\prime}, \ldots, \mathbf{q}_{k_{2}}^{\prime}, \ldots, \mathbf{q}_{k_{s-1}+1}^{\prime}, \ldots, \mathbf{q}_{k_{s}}^{\prime}\right),
$$

$\mathbf{q}_{k}^{\prime}=\left(x_{k}^{\prime}, y_{k}^{\prime}, z_{k}^{\prime}, w_{k}^{\prime}\right)$, where we have grouped the equal terms $\mathbf{q}_{1}^{\prime}=$ $\ldots=\mathbf{q}_{k_{1}}^{\prime}, \mathbf{q}_{k_{1}+1}^{\prime}=\ldots=\mathbf{q}_{k_{2}}^{\prime}, \ldots, \mathbf{q}_{k_{s-1}+1}^{\prime}=\ldots=\mathbf{q}_{k_{s}}^{\prime}, k_{s}=n$. We call each group of particles a cluster of $X$. Denote by $\Lambda_{i}$ the index set of the $i$-th cluster, i.e., $\Lambda_{i}=\left\{k_{i-1}+1, \ldots, k_{i}\right\}, 1 \leq i \leq s$. Let $\left|\Lambda_{1}\right|=k_{1}, \ldots,\left|\Lambda_{i}\right|=$ $k_{i}-k_{i-1}, \ldots,\left|\Lambda_{s}\right|=n-k_{s-1}$. Let $\mathbf{q}(l)=\left(\mathbf{q}_{1(l)}, \ldots, \mathbf{q}_{n(l)}\right), l=1, \ldots, \infty$, be a sequence of ordinary central configurations that converges to $X$. Let $\mathbf{q}_{i(l)}=\left(x_{i(l)}, y_{i(l)}, z_{i(l)}, w_{i(l)}\right)$. Denote by $\lambda(l)$ the multiplier of $\mathbf{q}(l)$.

Proposition 4. Given $n$ masses in $\mathbb{H}^{3}$, if there is a sequence of ordinary central configurations that converges to some point $X \in \Delta_{-}$, then the sequence of multipliers approaches $-\infty$.

The set of ordinary central configurations is not compact. For example, consider the following regular polygonal configuration formed by $n$ equal masses

$$
\mathbf{q}_{i}=\left(\sinh \theta \cos \frac{i 2 \pi}{n}, \sinh \theta \cos \frac{i 2 \pi}{n}, 0, \cosh \theta\right), i=1, \ldots, n .
$$

It is easy to check that it is an ordinary central configuration for any $\theta \in(0, \infty)$. These ordinary central configurations are not in one class since there is no homothety symmetry in the set of ordinary central configurations. As $\theta \rightarrow 0$, the configuration converges to a singular configuration. Note that the momentum of inertia of that singular point is 0 .

Theorem 5. Given $n$ masses in $\mathbb{H}^{3}$ and any point $X \in \Delta_{-}$with $I_{-1}(X)=c>0$, there is a neighborhood of $X$ in which there is no ordinary central configuration. 
Remark 6. Consider the subset of ordinary central configurations on $\mathbb{H}_{x y w}^{2}$, the intersection of $\mathbb{H}^{3}$ and the hyperplane $z=0$, with the property that all particles lie on a same plane perpendicular to the $w$-axis and the value of the multiplier is fixed. Tibboel [24] proved that this subset is compact in the configuration space. Our result is stronger.

In $\mathbb{S}^{3}$, a point in $\Delta_{+}$can be written as

$$
X=\left(\mathbf{q}_{1}^{\prime}, \ldots, \mathbf{q}_{k_{1}}^{\prime}, \mathbf{q}_{k_{1}+1}^{\prime}, \ldots, \mathbf{q}_{k_{2}}^{\prime}, \ldots, \mathbf{q}_{k_{2 s-1}+1}^{\prime}, \ldots, \mathbf{q}_{k_{2 s}}^{\prime}\right)
$$

where we have grouped the equal and antipodal terms $\mathbf{q}_{1}^{\prime}=\ldots=$ $\mathbf{q}_{k_{1}}^{\prime}, \mathbf{q}_{k_{1}+1}^{\prime}=\ldots=\mathbf{q}_{k_{2}}^{\prime},-\mathbf{q}_{k 1}^{\prime}=\mathbf{q}_{k_{2}}^{\prime}, \ldots, \mathbf{q}_{k_{2 s-1}+1}^{\prime}=\ldots=\mathbf{q}_{k_{2 s}}^{\prime},-\mathbf{q}_{k_{2 s-1}}=$ $\mathbf{q}_{k_{2 s}}^{\prime}, k_{2 s}=n$. If $\left|\Lambda_{k}\right|>1$, particles in the $k$-th cluster form a collision singular configuration. If $\left|\Lambda_{2 i-1}\right|=\left|\Lambda_{2 i}\right|=1$, particles in the two cluster form an antipodal singular configuration. If $\left|\Lambda_{2 i-1}\right| \geq 2$ and $\left|\Lambda_{2 i}\right| \geq 1$, particles in the two cluster form a collision-antipodal singular configuration.

As in the case of $\mathbb{H}^{3}$, the set of ordinary central configurations is not compact. For instance, consider the regular polygonal configurations formed by $n$ equal masses at position

$$
\mathbf{q}_{i}=\left(\sin \theta \cos \frac{i 2 \pi}{n}, \sin \theta \cos \frac{i 2 \pi}{n}, \cos \theta, 0\right), i=1, \ldots, n .
$$

They approach a collision singular configuration with momentum of inertia 0 as $\theta \rightarrow 0$. The situation is more complicated than that in $\mathbb{H}^{3}$. By the transform $\tau \in O(4)$ of Theorem 10, we get a 1-parameter family of ordinary central configurations approaching a singular point with momentum of inertia $n$. Another examples, consider three masses $m_{1}=m, m_{2}=m_{3}=M$ at position

$$
\mathbf{q}_{1}=(1,0,0,0), \mathbf{q}_{2}=(-\cos \theta, 0, \sin \theta, 0), \mathbf{q}_{3}=(-\cos \theta, 0,-\sin \theta, 0) \text {. }
$$

It is an ordinary central configuration for any $\theta \neq 0$ by equation (5). As $\theta \rightarrow 0$, the configurations approaches a collision-antipodal singular configuration. Let $\mathbb{S}_{x y}^{1}:=\left\{(x, y, z, w) \in \mathbb{S}^{3}: z=w=0\right\}, \mathbb{S}_{z w}^{1}:=$ $\left\{(x, y, z, w) \in \mathbb{S}^{3}: x=y=0\right\}$. Note that the singular configurations of the three examples all lie on the union of two circles, $\mathbb{S}_{x y}^{1} \cup \mathbb{S}_{z w}^{1}$.

We consider only a subset, denoted by $\mathcal{A}$, of $\Delta_{+}$with the following two properties: 1 . if $X \in \mathcal{A}$, then not all particles of $X$ lie on $\mathbb{S}_{x y}^{1} \cup \mathbb{S}_{z w}^{1}$; 2. $X$ contains collision singular sub configuration or antipodal singular sub configuration, that is, there is some $i$ such that $\left|\Lambda_{2 i-1}\right| \geq 2,\left|\Lambda_{2 i}\right|=$ 0 , or, $\left|\Lambda_{2 i-1}\right|=\left|\Lambda_{2 i}\right|=1$.

Theorem 7. Given $n$ masses in $\mathbb{S}^{3}$ and any point $X \in \mathcal{A}$, there is a neighborhood of $X$ in which there is no ordinary central configuration. 
We now consider the geodesic configurations. Let us introduce some notations. A geodesic central configuration is one for which all particles lie on a same geodesic. A 2-dimensional central configuration is one for which all particles lie on a same 2-dimensional great sphere but not on a same geodesic. Denote by $\mathbb{S}_{x y z}^{2}\left(\right.$ resp. $\left.\mathbb{S}_{x z w}^{2}\right)$ the 2-dimensional great sphere intersected by $\mathbb{S}^{3}$ and the hyperplane $w=0$ (resp. $\left.y=0\right)$. Denote by $\mathbb{H}_{x y w}^{2}$ the intersection of $\mathbb{H}^{3}$ and the hyperplane $z=0$. Let $\mathbb{S}_{x z}^{1}:=\left\{(x, y, z, w) \in \mathbb{S}^{3}: y=w=0\right\}, \mathbb{H}_{x w}^{1}:=\left\{(x, y, z, w) \in \mathbb{H}^{3}: y=\right.$ $z=0\}$. We have some related preliminary results.

Theorem 8 ([9]). Let $\mathbf{q}=\left(\mathbf{q}_{1}, \ldots, \mathbf{q}_{n}\right), \mathbf{q}_{i}=\left(x_{i}, y_{i}, z_{i}, w_{i}\right), \quad i=$ $1, \ldots, n$, be an ordinary central configuration in $\mathbb{H}^{3}\left(\mathbb{S}^{3}\right)$. Then we have the relationships

$$
\sum_{i=1}^{n} m_{i} x_{i} z_{i}=\sum_{i=1}^{n} m_{i} x_{i} w_{i}=\sum_{i=1}^{n} m_{i} y_{i} z_{i}=\sum_{i=1}^{n} m_{i} y_{i} w_{i}=0 .
$$

Remark 9. The above relationships have been found in $[11,12]$ for two-body ordinary central configurations, where it reads as $m_{1} \sin 2 \theta_{1}=$ $m_{2} \sin 2 \theta_{2}$ or $m_{1} \sinh 2 \theta_{1}=m_{2} \sinh 2 \theta_{2}$. Recall that configurations of relative equilibrium in $\mathbb{R}^{2}$ have center of mass at the origin, i.e., $\sum_{i=1}^{n} m_{i} x_{i}=\sum_{i=1}^{n} m_{i} y_{i}=0$. Theorem 8 can be viewed as an analogy of that fact.

Theorem $10([9])$. In $\mathbb{S}^{3}$, each geodesic (resp. 2-dimensional) ordinary central configuration is equivalent to one on $\mathbb{S}_{x z}^{1}$ (resp. $\mathbb{S}_{x y z}^{2}$ or $\left.\mathbb{S}_{x z w}^{2}\right)$. Any ordinary central configuration with multiplier $\lambda$ is mapped to one ordinary central configuration with multiplier $-\lambda$ by $\tau \in O(4)$, where $\tau(x, y, z, w)=(z, w, x, y)$.

Theorem 11 ([9, 27]). In $\mathbb{H}^{3}$, each ordinary central configuration is equivalent to one on $\mathbb{H}_{x y w}^{2}$. Each geodesic ordinary central configuration is equivalent to one on $\mathbb{H}_{x w}^{1}$.

Thus, for geodesic (resp. 2-dimensional ) ordinary central configurations, it is enough to study the ones on $\mathbb{S}_{x z}^{1}$ and $\mathbb{H}_{x w}^{1}$ (resp. $\mathbb{S}_{x y z}^{2}$ and $\mathbb{H}_{x y w}^{2}$ ). We will call the those special sub manifolds $\mathbb{S}^{1}$ and $\mathbb{H}^{1}$ (resp. $\mathbb{S}^{2}$ and $\mathbb{H}^{2}$ ).

The ordinary central configurations on $\mathbb{S}^{2}$ (resp. $\mathbb{H}^{2}$ ) are critical points of $U_{1}(\mathbf{q})$ (resp. $U_{-1}(\mathbf{q})$ ) restricted on $S_{c}^{+} \cap\left(\mathbb{S}^{2}\right)^{n}$ (resp. $S_{c}^{-} \cap$ $\left.\left(\mathbb{S}^{2}\right)^{n}\right)$. With a slight abuse of notation, we still call them $S_{c}^{+}$and $S_{c}^{-}$. Further more, the classes of ordinary central configurations in $I_{1}^{-1}(c)$ (resp. $\left.I_{-1}^{-1}(c)\right)$ correspond in 1-1 manner to the critical points of $U_{1}(\mathbf{q})$ (resp. $\left.U_{-1}(\mathbf{q})\right)$ restricted on $S_{c}^{+} / S^{1}$ (resp. $\left.S_{c}^{-} / S^{1}\right)$. Recall that for the 
Newtonian $n$-body problem, the classes of configurations of relative equilibrium correspond in 1-1 manner to the critical points of $U_{0}(\mathbf{q})$ restricted on $\mathcal{S} / S^{1}$, where

$$
\mathcal{S}=\left\{\mathbf{q} \in\left(\mathbb{R}^{2}\right)^{n}-\Delta \mid I_{0}(\mathbf{q})=1\right\} .
$$

For the $\mathbb{H}^{2}$ case, the set $S_{c}^{-} / S^{1}(c>0)$ is obviously diffeomorphic to $\mathcal{S} / S^{1}$. Thus, $S_{c}^{-} / S^{1}$ is smooth $(2 n-2)$-dimensional manifold.

Theorem 12. Given $n$ masses on $\mathbb{H}^{1}$ and any positive value of $c$, there are exactly $n ! / 2$ geodesic ordinary central configurations in $S_{c}^{-}$, one for each ordering of the masses along $\mathbb{H}^{1}$. At each of them, the Hessian of $\left.U_{-1}\right|_{S_{c}^{-} / S^{1}}$ has inertia $\left(n_{0}, n_{+}, n_{-}\right)=(0, n, n-2)$.

For the $\mathbb{S}^{2}$ case, the set $S_{c}^{+} / S^{1}$ is more complicated. Assume that $m_{1}$ is the smallest mass.

Proposition 13. For given $n$ masses, the critical values of the function $I_{1}(\mathbf{q})$ are $\left\{\sum \epsilon_{i} m_{i} \mid \epsilon_{i}=0\right.$ or 1$\}$. Assume that $m_{1}$ is the smallest mass. If $c<m_{1}$, then $S_{c}^{+}$is a smooth $(2 n-2)$-dimensional manifold with $2^{n}$ components.

Proof. Let $f(x, y, z)=x^{2}+y^{2},(x, y, z) \in \mathbb{S}^{2}$. Obviously, the critical points of $f$ consist of the equator and the two poles. Thus, the critical points of $I_{1}(\mathbf{q})$ are

$$
\left\{\mathbf{q} \in\left(\mathbb{S}^{2}\right)^{n} \mid \mathbf{q}_{i}=(0,0, \pm 1) \text { or }(\cos \varphi, \sin \varphi, 0), i=1, \ldots, n .\right\},
$$

which gives the set of critical values. If $c$ is less than the first critical value $m_{1}$, no particle of configurations in $S_{c}^{+}$can lie on the equator. Then each $z_{i}$ must be either positive or negative, which implies that there are $2^{n}$ components of $S_{c}^{+}$.

We will only consider geodesic ordinary central configurations on the following component of $S_{c}^{+}$,

$$
\mathcal{M}_{c}=\left\{\mathbf{q} \in S_{c}^{+} \mid z_{i}>0,1=1, \ldots, n\right\}, 0<c<m_{1} .
$$

Note that $\mathcal{M}_{c} / S^{1}$ is diffeomorphic to $\mathcal{S} / S^{1}$. The multiplier of ordinary central configurations on $\mathcal{M}_{c}$ must be negative, see Proposition 18.

Theorem 14. Given $n$ masses on $\mathbb{S}^{1}$ and any value of $c \in\left(0, \frac{m_{1}}{2}\right)$, there are exactly $n ! / 2$ geodesic ordinary central configurations on $\mathcal{M}_{c}$, one for each ordering of the masses. Provided that $0<c<\frac{m_{1}}{4}$, at each of them, the Hessian of $\left.U_{1}\right|_{\mathcal{M}_{c} / S^{1}}$ has inertia $\left(n_{0}, n_{+}, n_{-}\right)=(0, n, n-2)$.

The restriction of the value of $c$ might not be sharp, but it is necessary. In the two-body case, if $m_{1}<m_{2}$, then there is no ordinary central configuration on $\mathcal{M}_{c}$ for $c \in\left[m_{1}, m_{2}\right]$. If $m_{1}=m_{2}=m$, there 
is a continuum of ordinary central configurations on $\mathcal{M}_{m}$, and each of them has inertias $\left(n_{0}, n_{+}, n_{-}\right)=(1,1,0)$, see Section 10 of [9].

Recall that the inertia of each collinear configuration of relative equilibrium of the Newtonian n-body problem is also $\left(n_{0}, n_{+}, n_{-}\right)=$ $(0, n, n-2)$ if we study the Hessian of $U_{0}$ on $\left\{\mathbf{q}: I_{0}(\mathbf{q})=1\right\} / S^{1}$ instead of $\left\{\mathbf{q}: I_{0}(\mathbf{q})=1, \sum m_{i} \mathbf{q}_{i}=0\right\} / S^{1},[14]$. So Theorem 12 and 14 confirm the following general belief, namely, many properties of configurations of relative equilibrium of the Newtonian n-body problem also hold for ordinary central configurations of the curved n-body problem provided that the value of $I_{ \pm 1}(\mathbf{q})$ is small enough. In this particular case, i.e., the number and inertia of geodesic ordinary central configurations, Theorem 12 indicates that the restriction of small value of $I_{-1}(\mathbf{q})$ is not necessary on $\mathbb{H}^{2}$; Theorem 14 gives an upper bound of the value of $I_{1}(\mathbf{q})$ such that the corresponding results hold on $\mathbb{S}^{2}$.

The three manifolds $\mathcal{S} / S^{1}, S_{c}^{-} / S^{1}$ and $\mathcal{M}_{c} / S^{1}\left(0<c<m_{1}\right)$ are diffeomorphic, so share the same Poincaré polynomial. The number of geodesic ordinary central configurations on $\mathcal{M}_{c} / S^{1}\left(S_{c}^{-} / S^{1}\right)$ and Morse index of them are the same as that on $\mathcal{S} / S^{1}$ of the Newtonian $n$-body problem. By Theorem 5 and 7 , the set of ordinary central configurations on $\mathcal{M}_{c} / S^{1}\left(S_{c}^{-} / S^{1}\right)$ are compact. Thus, we can apply the argument of Palmore $[18,23]$ to obtain the following estimation on the number of critical points of $U_{1}(\mathbf{q})$ (resp. $U_{-1}(\mathbf{q})$ ) restricted on $\mathcal{M}_{c}$ (resp. $S_{c}^{-}$).

Corollary 15. Suppose that for a certain choice of masses of the curved $n$-body problem on $\mathbb{S}^{2}$ (resp. $\mathbb{H}^{2}$ ) all ordinary central configurations are non-degenerate critical points of $\left.U_{1}\right|_{\mathcal{M}_{c} / S^{1}}$ (resp. $\left.\left.U_{-1}\right|_{S_{c}^{-} / S^{1}}\right)$. Then in $\mathcal{M}_{c}\left(0<c<\frac{m_{1}}{4}\right)$ (resp. $\left.S_{c}^{-}(c>0)\right)$, there are at least $\frac{(3 n-4)(n-1) !}{2}$

ordinary central configurations, of which at least $\frac{(2 n-4)(n-1) !}{2}$ are nongeodesic.

\section{PROOF OF ThEOREM 5 AND ThEOREM 7}

Theorem 5 and 7 are analogous to Shub's lemma in the Newtonian $n$-body problem. It is first proved by Shub [21]. Moeckel gives a shorter proof in [14]. The idea of Moeckel is applicable if we use the following Cartesian coordinate system for $\left(\mathbb{S}^{3}\right)^{n}\left(\left(\mathbb{H}^{3}\right)^{n}\right)$.

Recall that we have written the Euler-Lagrange equation in Section 2.2 with the Cartesian coordinates. For each particle, we use the four coordinates $\left(x_{i}, y_{i}, z_{i}, w_{i}\right)$ to represent its position. That coordinate system is redundant. Three coordinates are enough to represent the positions of each particle. For instance, if $x_{i} \neq 0$, then $\mathbf{q}_{i}$ is in an 
open region of $\mathbb{S}^{3}$ for that $(y, z, w)$ can serve as a local chart. Then $\frac{\partial\left(U_{1}-\lambda I_{1}\right)}{\partial \mathbf{q}_{i}}=0$ is equivalent to $\frac{\partial\left(U_{1}-\lambda I_{1}\right)}{\partial y_{i}}=\frac{\partial\left(U_{1}-\lambda I_{1}\right)}{\partial z_{i}}=\frac{\partial\left(U_{1}-\lambda I_{1}\right)}{\partial w_{i}}=0$. Since $x_{i}^{2}+y_{i}^{2}=1-z_{i}^{2}-w_{i}^{2}$, so we have

$$
\frac{\partial I}{\partial y_{i}}=0, \frac{\partial I}{\partial z_{i}}=-2 m_{i} z_{i}, \frac{\partial I}{\partial w_{i}}=-2 m_{i} w_{i}
$$

By $\cos d_{i j}=x_{i} x_{j}+y_{i} y_{j}+z_{i} z_{j}+w_{i} w_{j}$, we obtain

$$
\frac{\partial \cot d_{i j}}{\partial y_{i}}=\frac{-1}{\sin ^{2} d_{i j}} \frac{\partial d_{i j}}{\partial y_{i}}=\frac{1}{\sin ^{3} d_{i j}}\left(\frac{\partial x_{i}}{\partial y_{i}} x_{j}+y_{j}\right)=\frac{1}{\sin ^{3} d_{i j}}\left(y_{j}-\frac{x_{j}}{x_{i}} y_{i}\right) \text {. }
$$

Similarly, we have $\frac{\partial \cot d_{i j}}{\partial z_{i}}=\frac{1}{\sin ^{3} d_{i j}}\left(z_{j}-\frac{x_{j}}{x_{i}} z_{i}\right), \frac{\partial \cot d_{i j}}{\partial w_{i}}=\frac{1}{\sin ^{3} d_{i j}}\left(w_{j}-\frac{x_{j}}{x_{i}} w_{i}\right)$. Thus, the central configuration equations for $\mathbf{q}_{i}$ can be written as

$$
\sum_{j \neq i} m_{i} m_{j} \frac{\mathbf{v}_{j}-\frac{x_{j}}{x_{i}} \mathbf{v}_{i}}{\sin ^{3} d_{i j}}=-\lambda 2 m_{i}\left(0, z_{i}, w_{i}\right), \quad \text { if } x_{i} \neq 0
$$

where $\mathbf{v}_{i}=\left(y_{i}, z_{i}, w_{i}\right)$. Similarly, if $w_{i} \neq 0$, the central configuration equation for $\mathbf{q}_{i}$ can be written as

$$
\sum_{j \neq i} m_{i} m_{j} \frac{\mathbf{u}_{j}-\frac{w_{j}}{w_{i}} \mathbf{u}_{i}}{\sin ^{3} d_{i j}}=\lambda 2 m_{i}\left(x_{i}, y_{i}, 0\right), \quad \text { if } w_{i} \neq 0,
$$

where $\mathbf{u}_{i}=\left(x_{i}, y_{i}, z_{i}\right)$. Similarly, the equations can be written in other forms if $y_{i} \neq 0$ or $z_{i} \neq 0$. In $\mathbb{H}^{3},(x, y, z)$ serve as a global chart. Thus, the central configuration equations in $\mathbb{H}^{3}$ can be written as

$$
\sum_{j \neq i} m_{i} m_{j} \frac{\mathbf{u}_{j}-\frac{w_{j}}{w_{i}} \mathbf{u}_{i}}{\sinh ^{3} d_{i j}}=\lambda 2 m_{i}\left(x_{i}, y_{i}, 0\right), i=1, \ldots, n .
$$

Proof of Proposition 4. View the two sides of the central configuration equations (7) as vectors in $\mathbb{R}^{3}$, and multiply on both sides by $\mathbf{u}_{i}$. We obtain

$$
2 \lambda m_{i}\left(x_{i}^{2}+y_{i}^{2}\right)=\sum_{j \neq i} \frac{\mathbf{u}_{j} \cdot \mathbf{u}_{i}-\frac{w_{j}}{w_{i}}\left(w_{i}^{2}-1\right)}{\sinh ^{3} d_{i j}}=\sum_{j \neq i} \frac{\frac{w_{j}}{w_{i}}-\cosh d_{i j}}{\sinh ^{3} d_{i j}} .
$$

Assume that $\left|\Lambda_{1}\right|=k_{1} \geq 2$. Denote by $\mathbf{q}(l)$ the sequence of ordinary central configurations that converges to $X$. For each $l$, assume that $\mathbf{q}_{i(l)}$ of the first cluster has the biggest value of $w$, i.e., $w_{i(l)} \geq w_{j(l)}$ for any $1 \leq j(l) \leq k_{1}$. The above equality for $\mathbf{q}_{i(l)}$ is

$$
2 \lambda(l) m_{i(l)}\left(x_{i(l)}^{2}+y_{i(l)}^{2}\right)=\sum_{j(l) \neq i(l), j(l) \in \Lambda_{1}} \frac{\frac{w_{j(l)}}{w_{i(l)}}-\cosh d_{i j(l)}}{\sinh ^{3} d_{i j(l)}}+O(1) .
$$


As $l \rightarrow \infty$, each term in the above sum approaches $-\infty$ since

$$
\frac{\frac{w_{j(l)}}{w_{i(l)}}-\cosh d_{i j(l)}}{\sinh ^{3} d_{i j(l)}} \leq \frac{1-\cosh d_{i j(l)}}{\sinh ^{3} d_{i j(l)}}=\frac{-\frac{d_{i j(l)}^{2}}{2}+O\left(d_{i j(l)}^{4}\right)}{\sinh ^{3} d_{i j(l)}} \rightarrow-\infty .
$$

The value of $x_{i(l)}^{2}+y_{i(l)}^{2}$ is obviously bounded above. Hence, the multiplier of the sequence of ordinary central configurations approaches $-\infty$.

Proof of Theorem 5. Since $I_{-1}(X)=c>0$, not all clusters of $X$ are at $(0,0,0,1)$. Assume that the first cluster is not at $(0,0,0,1)$. Note that the central configuration equations can be written as $\sum_{j \neq i} \frac{w_{i} \mathbf{u}_{j}-w_{j} \mathbf{u}_{i}}{\sinh ^{3} d_{i j}}=$ $2 \lambda m_{i} w_{i}\left(x_{i}, y_{i}, 0\right), i=1, \ldots, n$. Then adding the equations corresponding to particles in the first cluster, we obtain

$$
\sum_{i \in \Lambda_{1}} \sum_{j \neq i} \frac{w_{i} \mathbf{u}_{j}-w_{j} \mathbf{u}_{i}}{\sinh ^{3} d_{i j}}=\sum_{i \in \Lambda_{1}} \sum_{j \notin \Lambda_{1}} \frac{w_{i} \mathbf{u}_{j}-w_{j} \mathbf{u}_{i}}{\sinh ^{3} d_{i j}}=2 \lambda \sum_{i \in \Lambda_{1}} m_{i} w_{i}\left(x_{i}, y_{i}, 0\right) .
$$

Assume that there is a sequence of ordinary central configurations $\mathbf{q}(l)$ that converges to $X$. The above equality reads $O(1)=\infty$. This contradiction shows that there is a neighborhood of $X$ in which there is no ordinary central configuration.

Proof of Theorem 7 . Let $X \in \mathcal{A}$. Assume that the first cluster of $X$ does not lie on $\mathbb{S}_{x y}^{1} \cup \mathbb{S}_{z w}^{1}$, i.e., $\mathbf{q}_{1}^{\prime} \notin \mathbb{S}_{x y}^{1} \cup \mathbb{S}_{z w}^{1}$. Since $z_{1}^{\prime 2}+w_{1}^{\prime 2} \neq 0$, we can assume that $w_{1}^{\prime} \neq 0$. Let $\mathbf{q}$ be an ordinary central configuration close to $X$. Then the central configuration equations for particles in the first two clusters can be written as

$$
\sum_{j \neq i, j \in \Lambda_{1} \cup \Lambda_{2}} \frac{w_{i} \mathbf{u}_{j}-w_{j} \mathbf{u}_{i}}{\sin ^{3} d_{i j}}+O(1)=2 \lambda m_{i} w_{i}\left(x_{i}, y_{i}, 0\right), i \in \Lambda_{1} \cup \Lambda_{2},
$$

where the $O(1)$ term corresponds to interactions between $\mathbf{q}_{i}$ and particles of the other $2 s-2$ clusters. Adding those equations, we obtain

$$
\sum_{i \in \Lambda_{1} \cup \Lambda_{2}}\left(\sum_{j \neq i, j \in \Lambda_{1} \cup \Lambda_{2}} \frac{w_{i} \mathbf{u}_{j}-w_{j} \mathbf{u}_{i}}{\sinh ^{3} d_{i j}}+O(1)\right)=O(1)=2 \lambda \sum_{i \in \Lambda_{1} \cup \Lambda_{2}} m_{i} w_{i}\left(x_{i}, y_{i}, 0\right) .
$$

Assume that there is a sequence of ordinary central configurations $\mathbf{q}(l)$ that converges to $X$. Since $X$ contains sub configuration which is collision singular or antipodal singular, we can prove that the absolute value of multiplier $|\lambda(l)|$ goes to infinite by argument similar to that in the proof of Proposition 4. Note that

$$
\sum_{i \in \Lambda_{1} \cup \Lambda_{2}} m_{i} w_{i}\left(x_{i}, y_{i}, 0\right) \rightarrow \sum_{i=1}^{k_{1}} m_{i}\left(x_{1}^{\prime} w_{1}^{\prime}, y_{1}^{\prime} w_{1}^{\prime}, 0\right)+\sum_{i=k_{1}+1}^{k_{2}} m_{i}\left(x_{1}^{\prime} w_{1}^{\prime}, y_{1}^{\prime} w_{1}^{\prime}, 0\right) \neq 0
$$


since $x_{1}^{\prime 2}+y_{1}^{\prime 2} \neq 0$ and $w_{1}^{\prime} \neq 0$. The above equality reads $O(1)=\infty$. This contradiction shows that there is a neighborhood of $X$ in which there is no ordinary central configuration.

\section{Proof of Theorem 12 And Theorem 14}

In the Newtonian $n$-body problem, the number of collinear configurations of relative equilibrium is first found to be $\frac{n !}{2}$ by Moulton [16], then Smale gives a shorter proof [22]. The index of them is $n-2$ [17]. The idea in [17], due to Conley, is applicable if we use an angle coordinate system for $\left(\mathbb{S}^{2}\right)^{n}\left(\left(\mathbb{H}^{2}\right)^{n}\right)$. Theorem 12 (the $\mathbb{H}^{2}$ case) is proved in the first subsection, divided into two parts. Theorem 14 (the $\mathbb{S}^{2}$ case) is proved by a similar way with minor modification in the last subsection.

\subsection{Geodesic ordinary central configurations on $\mathbb{H}^{2}$.}

Proposition 16. Let $\mathbf{q}$ be an ordinary central configuration on $\mathbb{H}^{2}$. Then the multiplier is negative.

Proof. Assume $w_{i} \leq w_{n}$ for $i=1, \ldots, n$. Then $w_{n}>1$. Recall that the central configuration equation (7) for $\mathbf{q}_{n}$ can be written as $\sum_{j \neq n} \frac{\mathbf{u}_{j}-\frac{w_{j}}{w_{n}} \mathbf{u}_{n}}{\sinh ^{3} d_{j n}}=2 \lambda m_{n} \mathbf{u}_{n}$. Here $\mathbf{u}_{i}=\left(x_{i}, y_{i}\right)$ since the configuration is on $\mathbb{H}^{2}$. Multiplying $\mathbf{u}_{n}$ on both sides, the right side becomes $2 \lambda m_{n}\left(w_{n}^{2}-1\right)$, and the left side becomes

$$
\sum_{j \neq n} \frac{\mathbf{u}_{j} \cdot \mathbf{u}_{n}-\frac{w_{j}}{w_{n}}\left(w_{n}^{2}-1\right)}{\sinh ^{3} d_{j n}}=\sum_{j \neq n} \frac{\frac{w_{j}}{w_{n}}-\cosh d_{j n}}{\sinh ^{3} d_{j n}}<0 .
$$

This shows that the multiplier $\lambda$ is negative.

We use an angle coordinate system for $\mathbb{H}^{2},(\theta, \varphi), \theta, \varphi \in \mathbb{R}$. The relationship between Cartesian coordinates $(x, y, w)$ and $(\theta, \varphi)$ is

$$
(x, y, w)=(\sinh \theta, \cosh \theta \sinh \varphi, \cosh \theta \cosh \varphi) .
$$

Then $\mathbb{H}^{1}=\mathbb{H}_{x w}^{1}$ is parameterized by $(\theta, 0)$ and $\left(\mathbb{H}^{2}\right)^{n}$ is parameterized by $\left(\theta_{1}, \ldots, \theta_{n}, \varphi_{1}, \ldots, \varphi_{n}\right)$. The momentum of inertia is

$$
I_{-1}(\mathbf{q})=\sum_{i=1}^{n} m_{i}\left(x_{i}^{2}+y_{i}^{2}\right)=\sum_{i=1}^{n} m_{i}\left(\sinh ^{2} \theta_{i}+\cosh ^{2} \theta_{i} \sinh ^{2} \varphi_{i}\right) .
$$

In the above angle coordinates, a configuration $\left(\theta_{1}, \ldots, \theta_{n}, \varphi_{1}, \ldots, \varphi_{n}\right)$ is an ordinary central configuration if

$$
\frac{\partial U_{-1}}{\partial \theta_{i}}=\lambda \frac{\partial I_{-1}}{\partial \theta_{i}}, \frac{\partial U_{-1}}{\partial \varphi_{i}}=\lambda \frac{\partial I_{-1}}{\partial \varphi_{i}}, i=1, \ldots, n .
$$


Restricted on $\mathbb{H}^{1}$, we can just use the theta coordinates $\left(\theta_{1}, \ldots, \theta_{n}\right)$ to parametrize the configurations. Then

$$
\begin{array}{lr}
\mathbf{q}_{i}=\left(\sinh \theta_{i}, 0, \cosh \theta_{i}\right), & d_{i j}=\left|\theta_{i}-\theta_{j}\right|, \\
U(\mathbf{q})=\sum_{1 \leq i<j \leq n} m_{i} m_{j} \operatorname{coth} d_{i j} & I(\mathbf{q})=\sum_{i=1}^{n} m_{i} \sinh ^{2} \theta_{i} .
\end{array}
$$

A configuration $\left(\theta_{1}, \ldots, \theta_{n}\right)$ is an ordinary central configuration if $\frac{\partial U_{-1}}{\partial \theta_{i}}=$ $\lambda \frac{\partial I_{-1}}{\partial \theta_{i}}$, or explicitly,

$$
\sum_{j \neq i} \frac{m_{i} m_{j} \sinh \left(\theta_{j}-\theta_{i}\right)}{\sinh ^{3} d_{i j}}=\lambda m_{i} \sinh 2 \theta_{i}, i=1, \ldots, n
$$

Proof of Theorem 12, Part I. The numbers of geodesic ordinary central configurations is proved to be $\frac{n !}{2}$ in [9]. The argument is similar to that of the Newtonian $n$-body problem [22]. We briefly repeat the idea here.

Restrict the function $U_{-1}$ on the set $S_{c}^{-} \cap\left(\mathbb{H}^{1}\right)^{n}=\left\{\mathbf{q} \in\left(\mathbb{H}^{1}\right)^{n}-\right.$ $\left.\Delta_{-} \mid I_{-1}(\mathbf{q})=c\right\}$, which has $n$ ! components. Each component is homeomorphic to an open ball. On each component, there is at least one minimum. All critical points are minima since the Hessian of $U_{-1}$ at each of them is positive definite. Thus, there are $n$ ! critical points, and the number of ordinary central configurations is $\frac{n !}{2}$ by the $S O(2)$ symmetry.

For completeness, we repeat the proof of positive definiteness. Let $\overline{\mathbf{q}}=\left(\bar{\theta}_{1}, \ldots, \bar{\theta}_{n}\right)$ be one critical point of $\left.U_{-1}\right|_{S_{c}^{-}}$. Assume that $\bar{\theta}_{1}<\ldots<$ $\bar{\theta}_{n}$ and that the multiplier is $\bar{\lambda}$. Then the Hessian of $\left.U_{-1}\right|_{S_{c}^{-} \cap\left(\mathbb{H}^{1}\right)^{n}}$ equals $\left.D^{2}\left(U_{-1}-\bar{\lambda} I_{-1}\right)\right|_{\overline{\mathbf{q}}}$ as quadratic forms on $T_{\overline{\mathbf{q}}} S_{c}^{-} \cap\left(\mathbb{H}^{1}\right)^{n}$, the tangent space of $S_{c}^{-} \cap\left(\mathbb{H}^{1}\right)^{n}$ at $\overline{\mathbf{q}}$. By straightforward computations, we obtain

$$
\begin{aligned}
&\left.D^{2}\left(U_{-1}-\bar{\lambda} I_{-1}\right)\right|_{\overline{\mathbf{q}}} \\
&=2\left[\begin{array}{cccc}
\sum_{j=1, j \neq 1}^{n} \frac{m_{1} m_{j} \cosh d_{1 j}}{\sinh ^{3} d_{1 j}} & -\frac{m_{1} m_{2} \cosh d_{12}}{\sinh ^{3} d_{12}} & \ldots & -\frac{m_{1} m_{n} \cosh d_{1 n}}{\sinh ^{3} d_{1 n}} \\
-\frac{m_{2} m_{1} \cosh d_{12}}{\sinh ^{3} d_{12}} & \sum_{j=1, j \neq 2}^{n} \frac{m_{2} m_{j} \cosh d_{2 j}}{\sinh ^{3} d_{2 j}} & \ldots & -\frac{m_{2} m_{n} \cosh d_{2 n}}{\sinh ^{3} d_{2 n}} \\
\ldots & \ldots & \ldots & \ldots \\
-\frac{m_{1} m_{n} \cosh d_{1 n}}{\sinh ^{3} d_{1 n}} & \cdots & \ldots & \sum_{j=1, j \neq n}^{n} \frac{m_{n} m_{j} \cosh d_{n j}}{\sinh ^{3} d_{n j}}
\end{array}\right]
\end{aligned}
$$




$$
-2 \bar{\lambda}\left[\begin{array}{cccc}
m_{1} \cosh 2 \theta_{1} & 0 & \cdots & 0 \\
0 & m_{2} \cosh 2 \theta_{2} & \cdots & 0 \\
\cdots & \cdots & \cdots & \cdots \\
0 & \cdots & \cdots & m_{n} \cosh 2 \theta_{n}
\end{array}\right] .
$$

The second part is obviously positive definite. For the first part, let us take any nonzero vector $\mathbf{v}=\left(v_{1}, \cdots, v_{n}\right) \in T_{\overline{\mathbf{q}}} S_{c}^{-} \cap\left(\mathbb{H}^{1}\right)^{n}$. We obtain

$$
\begin{aligned}
\mathbf{v}^{T}\left(D^{2} U_{-1}\right) \mathbf{v} & =\sum_{i=1}^{n} \sum_{j=1}^{n}\left(D^{2} U_{-1}\right)_{i j} v_{i} v_{j}=2 \sum_{i=1}^{n} \sum_{\substack{j=1 \\
j \neq i}}^{n} \frac{m_{i} m_{j} \cosh d_{i j}}{\sinh ^{3} d_{i j}} v_{i}^{2} \\
& -2 \sum_{i=1}^{n} \sum_{\substack{j=1 \\
j \neq i}}^{n} \frac{m_{i} m_{j} \cosh d_{i j}}{\sinh ^{3} d_{i j}} v_{i} v_{j}=\sum_{i=1}^{n} \sum_{\substack{j=1 \\
j \neq i}}^{n} \frac{m_{i} m_{j} \cosh d_{i j}}{\sinh ^{3} d_{i j}}\left(v_{i}-v_{j}\right)^{2} \geq 0 .
\end{aligned}
$$

Hence, we conclude that Hessian of $\left.U_{-1}\right|_{S_{c}^{-} \cap\left(\mathbb{H}^{1}\right)^{n}}$ is positive definite.

We have seen that system (8) have $n ! / 2$ solutions. Each is a minimum of $\left.U_{-1}\right|_{S_{c}^{-} \cap\left(\mathbb{H}^{1}\right)^{n}}$. We are going to study the Hessian on $S_{c}^{-}$at those critical points.

Let $\overline{\mathbf{q}}=\left(\bar{\theta}_{1}, \ldots, \bar{\theta}_{n}, 0, \ldots, 0\right)$ be one of the $n ! / 2$ geodesic ordinary central configurations. Assume that $\bar{\theta}_{1}<\ldots<\bar{\theta}_{n}$ and that the multiplier is $\bar{\lambda}$. Then $\overline{\mathbf{q}}$ is also a critical point of $f=U_{-1}-\bar{\lambda} I_{-1}$. Denote by $\mathcal{H}\left(\left.U_{-1}\right|_{S_{c}^{-}}, \overline{\mathbf{q}}\right)$ (resp. $\left.\mathcal{H}(f, \overline{\mathbf{q}})\right)$ the Hessian of $\left.U_{-1}\right|_{S_{c}^{-}}$(resp. $f$ ) at the critical point $\overline{\mathbf{q}}$. Then we have

$$
\mathcal{H}(f, \overline{\mathbf{q}})=\mathcal{H}\left(\left.U_{-1}\right|_{S_{c}^{-}}, \overline{\mathbf{q}}\right)
$$

as quadratic forms on $T_{\overline{\mathbf{q}}} S_{c}^{-}$, the tangent space of $S_{c}^{-}$at $\overline{\mathbf{q}}$.

The set $S_{c}^{-}$is hyper surface in $\left(\mathbb{H}^{2}\right)^{n}$ with co-dimension 1 . The tangent space of $\left(\mathbb{H}^{2}\right)^{n}$ at $\overline{\mathbf{q}}, T_{\overline{\mathbf{q}}}\left(\mathbb{H}^{2}\right)^{n}$, is spanned by $\frac{\partial}{\partial \theta_{1}}, \ldots, \frac{\partial}{\partial \theta_{n}}, \frac{\partial}{\partial \varphi_{1}}, \ldots, \frac{\partial}{\partial \varphi_{n}}$. The normal of $S_{c}^{-}$at $\overline{\mathbf{q}}$ is $\left.\nabla I_{-1}\right|_{\overline{\mathbf{q}}}=\sum_{i=1}^{n} m_{i} \sinh 2 \bar{\theta}_{i} \frac{\partial}{\partial \theta_{i}}$, which is in the subspace spanned by $\frac{\partial}{\partial \theta_{1}}, \ldots, \frac{\partial}{\partial \theta_{n}}$. Thus, we see $T_{\overline{\mathbf{q}}} S_{c}^{-}=V_{1} \oplus V_{2}$,

$$
V_{1}=<\frac{\partial}{\partial \theta_{1}}, \ldots, \frac{\partial}{\partial \theta_{n}}>/<\left.\nabla I_{-1}\right|_{\overline{\mathbf{q}}}>, V_{2}=<\frac{\partial}{\partial \varphi_{1}}, \ldots, \frac{\partial}{\partial \varphi_{n}}>.
$$

where $\left.<b_{1} \ldots, b_{n}\right\rangle$ is the linear space spanned by the vectors $b_{1}, \ldots, b_{n}$.

We claim that $\mathcal{H}(f, \overline{\mathbf{q}})$ is block-diagonal with respect to this splitting of $T_{\overline{\mathbf{q}}} S_{c}^{-}$. Direct computation leads to $\left.\frac{\partial^{2} I_{-1}}{\partial \theta_{i} \partial \varphi_{j}}\right|_{\overline{\mathbf{q}}}=0$ for all pairs of $(i, j)$. We claim that $\left.\frac{\partial^{2} U_{-1}}{\partial \theta_{i} \partial \varphi_{j}}\right|_{\overline{\mathbf{q}}}=0$ for all pairs of $(i, j)$. Denote by $\mathbf{q}+h_{i}$ the coordinate $\left(\theta_{1}, \ldots, \theta_{i}+h, \ldots, \theta_{n}, \varphi_{1}, \ldots, \varphi_{n}\right)$, by $\mathbf{q}+k_{j}$ the coordinate 


$$
\begin{aligned}
& \left(\theta_{1}, \ldots, \theta_{n}, \varphi_{1}, \ldots, \varphi_{j}+k, \ldots, \varphi_{n}\right) \text {. Then } \\
& \left.\frac{\partial^{2} U_{-1}}{\partial \varphi_{j} \partial \theta_{i}}\right|_{\overline{\mathbf{q}}}=\lim _{k \rightarrow 0} \frac{1}{2 k}\left(\left.\frac{\partial U_{-1}}{\partial \theta_{i}}\right|_{\overline{\mathbf{q}}+k_{j}}-\left.\frac{\partial U_{-1}}{\partial \theta_{i}}\right|_{\overline{\mathbf{q}}-k_{j}}\right) \\
& =\lim _{(h, k) \rightarrow(0,0)} \frac{1}{2 h k}\left(U_{-1}\left(\overline{\mathbf{q}}+k_{j}+h_{i}\right)-U_{-1}\left(\overline{\mathbf{q}}+k_{j}\right)-U_{-1}\left(\overline{\mathbf{q}}-k_{j}+h_{i}\right)+U_{-1}\left(\overline{\mathbf{q}}-k_{j}\right)\right) \\
& =0 .
\end{aligned}
$$

Here, we use the symmetry $U_{-1}\left(\overline{\mathbf{q}}+k_{j}\right)=U_{-1}\left(\overline{\mathbf{q}}-k_{j}\right), U_{-1}\left(\overline{\mathbf{q}}+k_{j}+h_{i}\right)=$ $U_{-1}\left(\overline{\mathbf{q}}-k_{j}+h_{i}\right)$. Thus, $\mathcal{H}(f, \overline{\mathbf{q}})$ is block-diagonal,

$\left.\mathcal{H}(f, \overline{\mathbf{q}})\right|_{\overline{\mathbf{q}}}=D^{2} U_{-1}-\bar{\lambda} D^{2} I_{-1}=\operatorname{diag}\left\{\left[\frac{\partial^{2} U}{\partial \theta_{i} \partial \theta_{j}}-\bar{\lambda} \frac{\partial^{2} I_{-1}}{\partial \theta_{i} \partial \theta_{j}}\right],\left[\frac{\partial^{2} U}{\partial \varphi_{i} \partial \varphi_{j}}-\bar{\lambda} \frac{\partial^{2} I_{-1}}{\partial \varphi_{i} \partial \varphi_{j}}\right]\right\}$

Denote by $\mathcal{H}_{1}, \mathcal{H}_{2}$ the two $n \times n$ blocks respectively. If $b \in T_{\overline{\mathbf{q}}} S_{c}^{-}$has decomposition $b=b_{1}+b_{2}$, then

$$
\mathcal{H}(f, \overline{\mathbf{q}})(b)=\mathcal{H}_{1}\left(b_{1}\right)+\mathcal{H}_{2}\left(b_{2}\right)
$$

We pass to find the inertia of $\mathcal{H}_{1}$ on $V_{1}$ and that of $\mathcal{H}_{2}$ on $V_{2}$. The first one has already been found in the above proof. Indeed, it is the Hessian of $\left.U_{-1}\right|_{S_{c}^{-} \cap\left(\mathbb{H}^{1}\right)^{n}}$ So $\mathcal{H}_{1}$ on $V_{1}$ has inertia $\left(n_{0}, n_{+}, n_{-}\right)=(0, n-1,0)$.

Proof of Theorem 12, Part II. By the above argument, it only remains to show that $\mathcal{H}_{2}$ on $V_{2}$ has inertia $\left(n_{0}, n_{+}, n_{-}\right)=(1,1, n-2)$.

Direct computation shows that $\mathcal{H}_{2}$ is

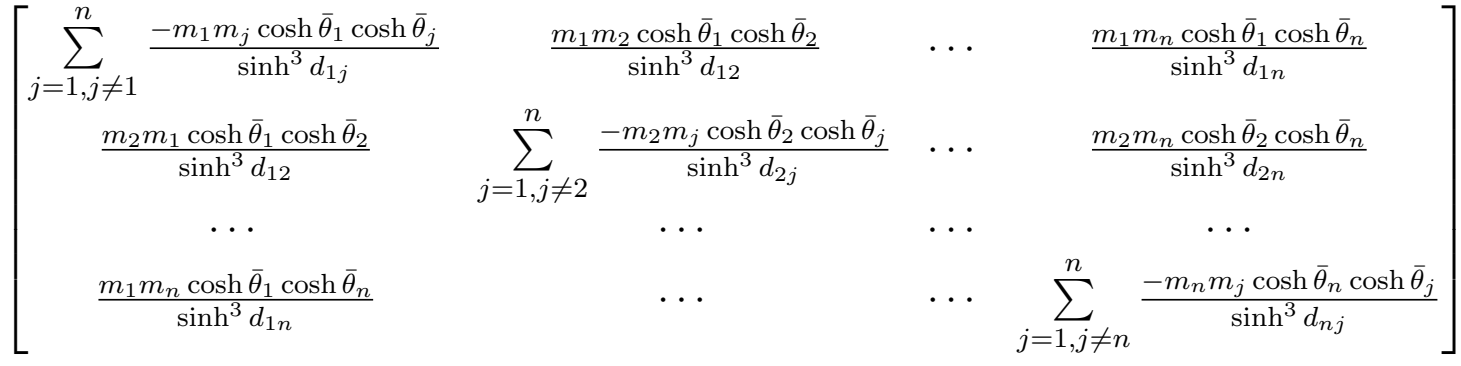

$$
\begin{aligned}
& -2 \bar{\lambda}\left[\begin{array}{cccc}
m_{1} \cosh ^{2} \bar{\theta}_{1} & 0 & \cdots & 0 \\
0 & m_{2} \cosh ^{2} \bar{\theta}_{2} & \cdots & 0 \\
\cdots & \cdots & \cdots & \cdots \\
0 & \cdots & \cdots & m_{n} \cosh ^{2} \bar{\theta}_{n}
\end{array}\right] \text {. }
\end{aligned}
$$


Let $C:=\operatorname{diag}\left\{\cosh \bar{\theta}_{1}, \cdots, \cosh \bar{\theta}_{n}\right\}, M:=\operatorname{diag}\left\{m_{1}, \cdots, m_{n}\right\}$, and

$$
A:=\left[\begin{array}{cccc}
\sum_{j=1, j \neq 1}^{n} \frac{-m_{j} \cosh \bar{\theta}_{j}}{\cosh \bar{\theta}_{1} \sinh ^{3} d_{1 j}} & \frac{m_{2}}{\sinh ^{3} d_{12}} & \cdots & \frac{m_{n}}{\sinh ^{3} d_{1 n}} \\
\frac{m_{1}}{\sinh ^{3} d_{12}} & \sum_{j=1, j \neq 2}^{n} \frac{-m_{j} \cosh \bar{\theta}_{j}}{\cosh \bar{\theta}_{2} \sinh ^{3} d_{2 j}} & \ldots & \frac{m_{n}}{\sinh ^{3} d_{2 n}} \\
\ldots & \ldots & \ldots & \ldots \\
\frac{m_{1}}{\sinh ^{3} d_{1 n}} & \ldots & \ldots & \sum_{j=1, j \neq n}^{n} \frac{-m_{j} \cosh _{j}}{\cosh \bar{\theta}_{n} \sinh ^{3} d_{n j}}
\end{array}\right] .
$$

Then it is easy to check that $\mathcal{H}_{2}=C M(A-2 \bar{\lambda}) C$. Let $D=C M$. Note that $C M, C, D$ are all positive definite and diagonal. Since

$$
\begin{aligned}
\mathcal{H}_{2} & =\left[\left(D^{\frac{1}{2}}\right)^{T} D^{\frac{1}{2}}(A-2 \bar{\lambda}) D^{\frac{-1}{2}} D^{\frac{1}{2}}\right] C \\
& =C^{\frac{1}{2}} C^{\frac{-1}{2}}\left[\left(D^{\frac{1}{2}}\right)^{T} D^{\frac{1}{2}}(A-2 \bar{\lambda}) D^{\frac{-1}{2}} D^{\frac{1}{2}}\right] C^{\frac{1}{2}}\left(C^{\frac{1}{2}}\right)^{T} .
\end{aligned}
$$

the inertia of $\mathcal{H}_{2}$ equals that of $A-2 \bar{\lambda}$ by Sylvester's law of inertia.

For the inertia of $A-2 \bar{\lambda}$, we will study the eigenvalues of $A$ and compare them with the negative constant $2 \bar{\lambda}$. First, there are two obvious eigenvectors of $A$ :

$$
\begin{array}{ll}
\mathbf{c}_{1}=\left(\cosh \bar{\theta}_{1}, \cdots, \cosh \bar{\theta}_{n}\right), & A \mathbf{c}_{1}=0 \mathbf{c}_{1}, \\
\mathbf{c}_{2}=\left(\sinh \bar{\theta}_{1}, \cdots, \sinh \bar{\theta}_{n}\right), & A \mathbf{c}_{2}=2 \bar{\lambda} \mathbf{c}_{2} .
\end{array}
$$

The first vector $\mathbf{c}_{1}$ can be obtained by inspecting the matrix, and the second vector $\mathbf{c}_{2}$ can be seen from equation (8).

Note that the matrix $C M A C$ is symmetric, sum of each row is zero, the diagonal is negative and other elements are positive. By the argument used in the proof of Theorem 12 (part I), we conclude that all other eigenvalues of $A$ is strictly negative.

We claim that all other eigenvalues of $A$ are smaller than $2 \bar{\lambda}$. The idea is to consider the linear vector field $Y=A \mathbf{u}$ in $\mathbb{R}^{n}, \mathbf{u}=\left(u_{1}, \cdots, u_{n}\right)^{T} \in$ $\mathbb{R}^{n}$. Introduce an inner product in $\mathbb{R}^{n},(\mathbf{u}, \mathbf{v})=\mathbf{u}^{T} M \mathbf{v}$. Then $A$ is symmetric with respect to this inner product and all eigenvectors of $A$ are mutually orthogonal. Then the line $t \mathbf{c}_{1}$ consists all fixed points of the flow, and the $(n-1)$-dimensional space $\mathbf{c}_{1}^{\perp}$ is invariant under the flow. In particular, each of the other eigenvectors corresponds to a stable manifold of the flow. Conley observed that to show that all other eigenvalues of $A$ are smaller than $2 \bar{\lambda}$ is equivalent to showing that the line $t \mathbf{c}_{2}$ attracts the flow lines. It is enough to find a cone $K \in \mathbf{c}_{1}^{\perp}$ around $t \mathbf{c}_{2}$ that is carried strictly inside itself by the flow. Define the 


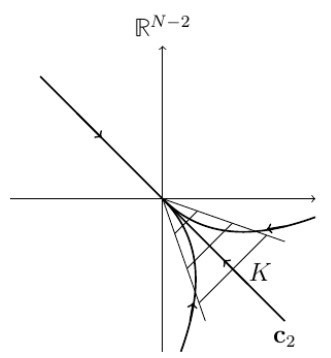

Figure 1. The linear flow in $\mathbf{c}_{1}^{\perp}$

polyhedral cone in $\mathbf{c}_{1}^{\perp}$ as

$K=\left\{\mathbf{u} \in \mathbb{R}^{n} \mid \sum_{i=1}^{n} m_{i} \cosh \bar{\theta}_{i} u_{i}=0, \frac{u_{1}}{\cosh \bar{\theta}_{1}} \leq \frac{u_{2}}{\cosh \bar{\theta}_{2}} \leq \cdots \leq \frac{u_{n}}{\cosh \bar{\theta}_{n}}\right\}$

We verify that $\mathbf{c}_{2} \in K$. First, $\mathbf{c}_{2} \perp \mathbf{c}_{1}$ since $\mathbf{c}_{2}$ is also an eigenvector of $A$. Or, the identity $\sum_{i=1}^{n} m_{i} x_{i} w_{i}=0$ of Theorem 8 reads $\left(\mathbf{c}_{1}, \mathbf{c}_{2}\right)=0$ in this case. Second, the inequalities in the definition read $\frac{\sinh \bar{\theta}_{1}}{\cosh \bar{\theta}_{1}} \leq$ $\frac{\sinh \bar{\theta}_{2}}{\cosh \bar{\theta}_{2}} \leq \cdots \leq \frac{\sinh \bar{\theta}_{n}}{\cosh \bar{\theta}_{n}}$, which is true since $\bar{\theta}_{1}<\bar{\theta}_{2}<\cdots<\bar{\theta}_{n}$.

We verify that $\mathbf{c}_{2}$ is the only eigenvector of $A$ in $K$. Since $\bar{\theta}_{1}<$ $\bar{\theta}_{2}<\cdots<\bar{\theta}_{n}$ and $\sum_{i=1}^{n} m_{i} \sinh 2 \bar{\theta}_{i}=0$, we assume that there is some $I \in(1, n)$ such that $\bar{\theta}_{1}<\ldots<\bar{\theta}_{I} \leq 0<\bar{\theta}_{I+1}<\ldots<\bar{\theta}_{n}$. Then by the definition of $K$, for any $\mathbf{u}=\left(u_{1}, \ldots, u_{n}\right)^{T} \in K$, we have

$$
\begin{cases}\frac{u_{i}}{\cosh \bar{\theta}_{i}} m_{i} \sinh 2 \bar{\theta}_{i} \geq \frac{u_{I}}{\cosh \bar{\theta}_{I}} m_{i} \sinh 2 \bar{\theta}_{i}, & 1 \leq i \leq I \\ \frac{u_{i}}{\cosh \bar{\theta}_{i}} m_{i} \sinh 2 \bar{\theta}_{i} \geq \frac{u_{I+1}}{\cosh \bar{\theta}_{I+1}} m_{i} \sinh 2 \bar{\theta}_{i}, \quad I+1 \leq i \leq n .\end{cases}
$$


Then for any point $\mathbf{u} \in K$, we have

$$
\begin{aligned}
2\left(\mathbf{u}, \mathbf{c}_{2}\right) & =2 \sum_{i=1}^{n} u_{i} m_{i} \sinh \bar{\theta}_{i}=\sum_{i=1}^{n} \frac{u_{i}}{\cosh \bar{\theta}_{i}} m_{i} \sinh 2 \bar{\theta}_{i} \\
& =\sum_{i=1}^{I} \frac{u_{i}}{\cosh \bar{\theta}_{i}} m_{i} \sinh 2 \bar{\theta}_{i}+\sum_{i=I+1}^{n} \frac{u_{i}}{\cosh \bar{\theta}_{i}} m_{i} \sinh 2 \bar{\theta}_{i} \\
& \geq \frac{u_{I}}{\cosh \bar{\theta}_{I}} \sum_{i=1}^{I} m_{i} \sinh 2 \bar{\theta}_{i}+\frac{u_{I+1}}{\cosh \bar{\theta}_{I+1}} \sum_{i=I+1}^{n} m_{i} \sinh 2 \bar{\theta}_{i} \\
& \geq\left(\frac{u_{I+1}}{\cosh \bar{\theta}_{I+1}}-\frac{u_{I}}{\cosh \bar{\theta}_{I}}\right) \sum_{i=I+1}^{n} m_{i} \sinh 2 \bar{\theta}_{i} \\
& \geq 0 .
\end{aligned}
$$

Moreover, the product is zero only if $\frac{u_{1}}{\cosh \bar{\theta}_{1}}=\cdots=\frac{u_{n}}{\cosh \bar{\theta}_{n}}$, which is impossible. Hence, $\left(\mathbf{u}, \mathbf{c}_{2}\right)>0$, and there is no other eigenvectors than $\mathbf{c}_{2}$ of $A$ in $K$.

The boundary $\partial K$ consists of points for which one or more equalities hold. However, except for the origin, at least one inequality must hold (otherwise $\mathbf{u}=t \mathbf{c}_{1}$ ). Consider a boundary point with

$$
\frac{u_{1}}{\cosh \bar{\theta}_{1}} \leq \cdots \frac{u_{i}}{\cosh \bar{\theta}_{i}}=\cdots=\frac{u_{j}}{\cosh \bar{\theta}_{j}} \leq \cdots \leq \frac{u_{n}}{\cosh \bar{\theta}_{n}}
$$

Let $g(\mathbf{u})=\frac{u_{j}}{\cosh \bar{\theta}_{j}}-\frac{u_{i}}{\cosh \bar{\theta}_{i}}$. Then $g=0$ at this point, and $g$ is positive in $K$. To prove that at this point the flow is pointing inwards, see Figure 1, we show $L_{Y} g=\frac{\dot{u}_{j}}{\cosh \bar{\theta}_{j}}-\frac{\dot{u}_{i}}{\cosh \bar{\theta}_{i}}>0$. Direct computation shows that $\frac{\dot{u}_{j}}{\cosh \bar{\theta}_{j}}-\frac{\dot{u}_{i}}{\cosh \bar{\theta}_{i}}$ is

$$
\begin{aligned}
& \sum_{k=1, k \neq j}^{n} \frac{m_{k}}{\cosh \bar{\theta}_{j} \sinh ^{3} d_{k j}}\left(u_{k}-\frac{u_{j} \cosh \bar{\theta}_{k}}{\cosh \bar{\theta}_{j}}\right)-\sum_{k=1, k \neq i}^{n} \frac{m_{k}}{\cosh \bar{\theta}_{i} \sinh ^{3} d_{k i}}\left(u_{k}-\frac{u_{i} \cosh \bar{\theta}_{k}}{\cosh \bar{\theta}_{i}}\right) \\
= & \sum_{k=1, k \neq i, j}^{n} m_{k}\left(\frac{u_{k}}{\sinh ^{3} d_{k j} \cosh \bar{\theta}_{j}}-\frac{u_{j} \cosh \bar{\theta}_{k}}{\cosh ^{2} \bar{\theta}_{j} \sinh ^{3} d_{k j}}-\frac{u_{k}}{\sinh ^{3} d_{k i} \cosh \bar{\theta}_{i}}+\frac{u_{i} \cosh \bar{\theta}_{k}}{\cosh ^{2} \bar{\theta}_{i} \sinh ^{3} d_{k i}}\right) \\
+ & \frac{m_{i}}{\sinh ^{3} d_{i j} \cosh \bar{\theta}_{j}}\left(u_{i}-\frac{u_{j} \cosh \bar{\theta}_{i}}{\cosh \bar{\theta}_{j}}\right)-\frac{m_{j}}{\sinh ^{3} d_{i j} \cosh \bar{\theta}_{i}}\left(u_{j}-\frac{u_{i} \cosh \bar{\theta}_{j}}{\cosh \bar{\theta}_{i}}\right) .
\end{aligned}
$$

Since $\frac{u_{i}}{\cosh \bar{\theta}_{i}}=\frac{u_{j}}{\cosh \bar{\theta}_{j}}$, the last two terms are zero, and the expression $\frac{u_{j} \cosh \bar{\theta}_{k}}{\cosh ^{2} \bar{\theta}_{j} \sinh ^{3} d_{k j}}$ in the first part can be written as $\frac{u_{i} \cosh \bar{\theta}_{k}}{\cosh \bar{\theta}_{i} \cosh \bar{\theta}_{j} \sinh ^{3} d_{k j}}$. Then 
$L_{Y} g$ can be written as

$$
\sum_{k=1, k \neq i, j}^{n} m_{k}\left(u_{k}-\frac{u_{i} \cosh \bar{\theta}_{k}}{\cosh \bar{\theta}_{i}}\right)\left(\frac{1}{\sinh ^{3} d_{k j} \cosh \bar{\theta}_{j}}-\frac{1}{\sinh ^{3} d_{k i} \cosh \bar{\theta}_{i}}\right) .
$$

Every term in this expression is non-negative by Proposition 17. Proposition 17 will be given after this proof:

(10)

$$
\left\{\begin{array}{l}
\text { If } k<i, \text { then } u_{k}-\frac{u_{i} \cosh \bar{\theta}_{k}}{\cosh \theta_{i}} \leq 0, \frac{1}{\sinh ^{3} d_{k j} \cosh \bar{\theta}_{j}}-\frac{1}{\sinh ^{3} d_{k i} \cosh \bar{\theta}_{i}}<0 . \\
\text { If } i \leq k \leq j, \text { then } u_{k}-\frac{u_{i} \cosh \bar{\theta}_{k}}{\cosh \bar{\theta}_{i}}=0 . \\
\text { If } j<k, \text { then } u_{k}-\frac{u_{i} \cosh \bar{\theta}_{k}}{\cosh \bar{\theta}_{i}} \geq 0, \frac{1}{\sinh ^{3} d_{k j} \cosh \bar{\theta}_{j}}-\frac{1}{\sinh ^{3} d_{k i} \cosh \bar{\theta}_{i}}>0 .
\end{array}\right.
$$

Moreover, at least one term is strictly positive since at least one inequality in the definition of the cone must hold. Thus, we have proved that on the boundary of the cone the flow is pointing inwards, or, all the other eigenvalues of $A$ are smaller than $2 \bar{\lambda}$.

Hence, the eigenvalues of $A-2 \bar{\lambda}$ are $-2 \bar{\lambda}>0,0, \lambda_{3}<0, \cdots, \lambda_{n}<0$. Then, the inertia of $A-2 \bar{\lambda}$ is $\left(n_{0}, n_{+}, n_{-}\right)=(1,1, n-2)$, so is the inertia of $\mathcal{H}_{2}$, on the space $V_{2}$. On the space $V_{2} / S^{1}$, obviously, the inertia is $\left(n_{0}, n_{+}, n_{-}\right)=(0,1, n-2)$. Combined with the inertia of $\mathcal{H}_{1}$, we conclude that the inertia of $\left.U_{-1}\right|_{S_{c}^{-} / S^{1}}$ is

$$
(0, n-1,0)+(0,1, n-2)=(0, n, n-2) .
$$

This completes the proof of Theorem 12 .

Proposition 17. If $\theta_{1}<\theta_{2}<\cdots<\theta_{n}$, then the following inequalities hold.

(1) If $k<i<j$, then $\sinh ^{3}\left(\theta_{j}-\theta_{k}\right) \cosh \theta_{j}-\sinh ^{3}\left(\theta_{i}-\theta_{k}\right) \cosh \theta_{i}>$ 0 .

(2) If $i<j<k$, then $\sinh ^{3}\left(\theta_{k}-\theta_{i}\right) \cosh \theta_{i}-\sinh ^{3}\left(\theta_{k}-\theta_{j}\right) \cosh \theta_{j}>$ 0 .

Proof. Let $h(x)=\sinh ^{3}\left(x-\theta_{k}\right) \cosh x-\sinh ^{3}\left(\theta_{i}-\theta_{k}\right) \cosh \theta_{i}$ defined for $x \geq \theta_{i}$. Then $h\left(\theta_{i}\right)=0$, and

$$
h^{\prime}(x)=\sinh ^{2}\left(x-\theta_{k}\right)\left[\cosh \left(2 x-\theta_{k}\right)+2 \cosh \left(x-\theta_{k}\right) \cosh x\right]>0 .
$$

This implies the first inequality. We omit the proof of the second one since it is similar.

4.2. The $\mathbb{S}^{2}$ case. We omit the proof of the following result since it is similar to that of Proposition 16.

Proposition 18. Let $\mathbf{q}$ be an ordinary central configuration on $\mathbb{S}^{2}$. If $z_{i}>0$ for all particles. Then the multiplier is negative. 
We use an angle coordinate system for $\left\{(x, y, z) \in \mathbb{S}^{2} \mid z>0\right\},(\theta, \varphi)$, with $-\frac{\pi}{2}<\theta<\frac{\pi}{2},-\frac{\pi}{2}<\varphi<\frac{\pi}{2}$. The relationship between Cartesian coordinates $(x, y, z)$ and $(\theta, \varphi)$ is

$$
(x, y, z)=(\sin \theta, \cos \theta \sin \varphi, \cos \theta \cos \varphi) .
$$

Then $\mathbb{S}^{1}=\mathbb{S}_{x z}^{1}$ is parameterized by $(\theta, 0)$ and $\left(\mathbb{S}^{2}\right)^{n}$ is parameterized by $\left(\theta_{1}, \ldots, \theta_{n}, \varphi_{1}, \ldots, \varphi_{n}\right)$, and the momentum of inertia is

$$
I_{1}(\mathbf{q})=\sum m_{i}\left(\sin ^{2} \theta+\cos ^{2} \theta \sin \varphi\right)
$$

Proof of Theorem 14. For the number of geodesic ordinary central configurations, we apply the argument used in the proof of Theorem 12 . All arguments run well except showing that the Hessian of $U_{1}$ restricted on $\mathcal{M}_{c} \cap\left(\mathbb{S}^{1}\right)^{n}$ at each critical point is positive definite. By direct computation, we obtain the Hessian

$$
\begin{gathered}
2\left[\begin{array}{ccccc}
\sum_{j=1, j \neq 1}^{n} \frac{m_{1} m_{j} \cos d_{1 j}}{\sin ^{3} d_{1 j}} & -\frac{m_{1} m_{2} \cos d_{12}}{\sin ^{3} d_{12}} & \cdots & -\frac{m_{1} m_{n} \cos d_{1 n}}{\sin ^{3} d_{1 n}} \\
-\frac{m_{2} m_{1} \cos d_{12}}{\sin ^{3} d_{12}} & \sum_{j=1, j \neq 2}^{n} \frac{m_{2} m_{j} \cos d_{2 j}}{\sin ^{3} d_{2 j}} & \cdots & -\frac{m_{2} m_{n} \cos d_{2 n}}{\sin ^{3} d_{2 n}} \\
\ldots & \cdots & \cdots & \ldots \\
-\frac{m_{1} m_{n} \cos d_{1 n}}{\sin ^{3} d_{1 n}} & & \cdots & \ldots & \sum_{j=1, j \neq n}^{n} \frac{m_{n} m_{j} \cos d_{n j}}{\sin ^{3} d_{n j}}
\end{array}\right] \\
-2 \lambda\left[\begin{array}{cccc}
m_{1} \cos 2 \theta_{1} & 0 & \cdots & 0 \\
0 & m_{2} \cos 2 \theta_{2} & \cdots & 0 \\
\cdots & \cdots & \cdots & \cdots \\
0 & \cdots & \cdots & m_{n} \cos 2 \theta_{n}
\end{array}\right] .
\end{gathered}
$$

Restricting $c<\frac{1}{2} m_{1}$. Then $m_{i} \sin ^{2} \theta<\frac{1}{2} m_{1},-\frac{\pi}{4}<\theta_{i}<\frac{\pi}{4}$ for all $i$. Hence, $\cos 2 \theta_{i}>0, d_{i j}<\frac{\pi}{2}$. The second part is positive definite since $\lambda<0$. Each element of the first matrix not on the diagonal is negative. By the argument used in the proof of Theorem 12 (part I), we see that the first matrix is positive semi-definite. Thus, the Hessian of $\left.U_{1}\right|_{\mathcal{M}_{c} \cap\left(\mathbb{S}^{1}\right)^{n}}$ is positive definite and there are exactly $n ! / 2$ geodesic ordinary central configurations on $\mathcal{M}_{c}$ provided $c<\frac{m_{1}}{2}$.

For the Morse index of the geodesic ordinary central configurations, we need to restrict further $c<\frac{1}{4} m_{1}$, which leads to $m_{i} \sin ^{2} \theta<\frac{1}{4} m_{1}$, $-\frac{\pi}{6}<\theta_{i}<\frac{\pi}{6}$ for all $i$. Then all the argument used in the proof of Theorem 12 works if we replace the hyperbolic functions with the trigonometrical ones. Especially, the inequalities (10) are replaced by the following inequalities.

Proposition 19. If $-\frac{\pi}{6}<\theta_{1}<\cdots<\theta_{n}<\frac{\pi}{6}$, then the following inequalities hold. 
(1) If $k<i<j$, then $\sin ^{3}\left(\theta_{j}-\theta_{k}\right) \cos \theta_{j}-\sin ^{3}\left(\theta_{i}-\theta_{k}\right) \cos \theta_{i}>0$.

(2) If $i<j<k$, then $\sin ^{3}\left(\theta_{k}-\theta_{i}\right) \cos \theta_{i}-\sin ^{3}\left(\theta_{k}-\theta_{j}\right) \cos \theta_{j}>0$.

Proof. We only prove the first inequality. Let $h(x)=\sin ^{3}\left(x-\theta_{k}\right) \cos x-$ $\sin ^{3}\left(\theta_{i}-\theta_{k}\right) \cos \theta_{i}$ defined for $x \geq \theta_{i}$. Then $h\left(\theta_{i}\right)=0$, and

$$
h^{\prime}(x)=\sin ^{2}\left(x-\theta_{k}\right)\left[\cos \left(2 x-\theta_{k}\right)+2 \cos \left(x-\theta_{k}\right) \cos x\right]>0 .
$$

This proves the first inequality.

This completes the proof of Theorem 14 .

\section{Appendix: The RELATIVE EQUILIBRIA AND CENTRAL CONFIGURATIONS}

Recall that $\mathbb{S}_{x y}^{1}:=\left\{(x, y, z, w) \in \mathbb{S}^{3}: z=w=0\right\}, \mathbb{S}_{z w}^{1}:=\{(x, y, z, w) \in$ $\left.\mathbb{S}^{3}: x=y=0\right\}$. Recall that the critical points of $U_{1}$ are special central configurations, the critical points of $U_{1}-\lambda I_{1}, U_{-1}-\lambda I_{-1}$ that are not special central configurations are ordinary central configurations. The motions in the form of $\mathbf{q}(t)=\mathbf{q}(0)$ are called equilibria, and the motions in the form of $\exp (t \xi) \mathbf{q}(\xi \neq 0)$ are called relative equilibria.

Proposition $20([9])$ - Let $\mathbf{q}=\left(\mathbf{q}_{1}, \ldots, \mathbf{q}_{n}\right) \in\left(\mathbb{H}^{3}\right)^{n}$ be an ordinary central configuration with multiplier $\lambda$. Then the associated relative equilibria are $B_{\alpha, \beta}(t) \mathbf{q}$ with $\alpha=\sqrt{-2 \lambda} \cos s, \beta=$ $\sqrt{-2 \lambda} \sin s, s \in(0,2 \pi]$.

- Let $\mathbf{q}=\left(\mathbf{q}_{1}, \ldots, \mathbf{q}_{n}\right) \in\left(\mathbb{S}^{3}\right)^{n}$ be a special central configuration. Then the associated equilibrium is $\mathbf{q}(t)=\mathbf{q}$. The associated relative equilibria are

- $A_{\alpha, \beta}(t) \mathbf{q}$ with $\alpha, \beta \in \mathbb{R}$ if all particles are on $\mathbb{S}_{x y}^{1} \cup \mathbb{S}_{z w}^{1}$;

- $A_{\alpha, \beta}(t) \mathbf{q}$ with $\beta= \pm \alpha, \alpha \in \mathbb{R}$, if not all particles are on $\mathbb{S}_{x y}^{1} \cup \mathbb{S}_{z w}^{1}$.

- Let $\mathbf{q}=\left(\mathbf{q}_{1}, \ldots, \mathbf{q}_{n}\right) \in\left(\mathbb{S}^{3}\right)^{n}$ be an ordinary central configuration. The associated relative equilibria are

$-A_{\alpha, \beta}(t) \mathbf{q}$ with $\alpha=\sqrt{2 \lambda} \sinh s, \beta=\sqrt{2 \lambda} \cosh s, s \in \mathbb{R}$, if $\lambda>0$

- $A_{\alpha, \beta}(t) \mathbf{q}$ with $\alpha=\sqrt{-2 \lambda} \cosh s, \beta=\sqrt{-2 \lambda} \sinh s, s \in \mathbb{R}$, if $\lambda<0$.

For one ordinary central configuration, among the set of associated relative equilibria, there are periodic ones (in $H^{3}, \beta=0$; in $S^{3}, \alpha+$ $k \beta=0$ for some $k \in \mathbb{Z}$ ), and quasi-periodic ones (in $H^{3}$, none; in $S^{3}$, $\alpha+k \beta \neq 0$ for any $k \in \mathbb{Z})$.

In Newtonian $n$-body problem, the relative equilibrium is always planar, while in curved $n$-body problem, the set of relative equilibria has richer structure. We divide them into three classes, the geodesic 
ones, the 2-dimensional ones, and the 3-dimensional ones. A geodesic relative equilibrium is one with all particles on the same geodesic for all $t$; a 2-dimensional relative equilibrium is one with all particles on the same 2-dimensional great sphere for all $t$ but not on a same geodesic; the others are 3-dimensional relative equilibria.

If a $k$-dimensional relative equilibrium is associated with a $m$-dimensional configuration, then $k \geq m$. Let $Q(t) \mathbf{q}$ be one relative equilibrium. Then it is a geodesic one if $\mathbf{q}$ is on a geodesic and $Q(t)$ keeps that geodesic; it is a 2-dimensional one if $\mathbf{q}$ is on a 2-dimensional great sphere (q may be a geodesic one in that sphere), and $Q(t)$ keeps that 2-dimensional great sphere.

Let $G_{1}=S O(2) \times S O^{+}(1,1)$ and $G_{2}=S O(2) \times S O(2)$. Assume that $\mathbf{q}$ is an ordinary central configuration in $\mathbb{H}^{3}$ (resp. $\mathbb{S}^{3}$ ). Then $g \mathbf{q}$ is an ordinary central configuration with the same multiplier if $g$ is in $G_{1}\left(\right.$ resp. $\left.G_{2}\right)$. If $B_{\alpha, \beta}(t) \mathbf{q}\left(\right.$ resp. $\left.A_{\alpha, \beta}(t) \mathbf{q}\right)$ are relative equilibria associated with $\mathbf{q}$, then the relative equilibria associated with $g \mathbf{q}$ are

$$
B_{\alpha, \beta}(t) g \mathbf{q}=g B_{\alpha, \beta}(t) \mathbf{q}, \quad\left(\operatorname{resp} . A_{\alpha, \beta}(t) g \mathbf{q}=g A_{\alpha, \beta}(t) \mathbf{q}\right) .
$$

Let $\tau$ be the isometry in $O(4), \tau(x, y, z, w)=(z, w, x, y)$. By Theorem 10 , if $\mathbf{q}$ is an ordinary central configuration in $\mathbb{S}^{3}$ with multiplier $\lambda$, then the multiplier of $\tau \mathbf{q}$ is $-\lambda$. If $A_{\alpha, \beta}(t) \mathbf{q}$ are relative equilibria associated with $\mathbf{q}$, then the solutions associated with $\tau \mathbf{q}$ are

$$
A_{\beta, \alpha}(t) \tau \mathbf{q}=\tau A_{\alpha, \beta}(t) \mathbf{q} .
$$

Thus, thanks to Theorem 10 and Theorem 11, to find geodesic and 2-dimensional relative equilibria, it is enough to assume that the associated ordinary central configuration lies on $\mathbb{H}_{x y w}^{2}$ for the $\mathbb{H}^{3}$ case, and on $\mathbb{S}_{x y z}^{2}$ with negative multiplier for the $\mathbb{S}^{3}$ case.

Proposition 21. Consider the curved $n$-body problem in $\mathbb{H}^{3}$. Let $G_{1}=$ $S O(2) \times S O^{+}(1,1)$.

- There is no geodesic relative equilibrium.

- Any 2-dimensional relative equilibria must be in one of the following three forms:

$-g B_{ \pm \sqrt{-2 \lambda}, 0}(t) \mathbf{q}$ and $g B_{0, \pm \sqrt{-2 \lambda}}(t) \mathbf{q}$ for $\mathbf{q}$ being a geodesic ordinary central configuration on $\mathbb{H}_{x w}^{1}$ with multiplier $\lambda$;

- $g B_{ \pm \sqrt{-2 \lambda}, 0}(t) \mathbf{q}$ for $\mathbf{q}$ being a 2-dimensional ordinary central configuration on $\mathbb{H}_{x y w}^{2}$ with multiplier $\lambda$, where $g$ is some isometry in $G_{1}$.

- Any other relative equilibrium is 3-dimensional.

Proof. Let $\mathbf{q}$ be an ordinary central configuration on $\mathbb{H}_{x w}^{1}$ with multiplier $\lambda$. The 1-parameter subgroup $B_{\alpha, \beta}(t)$ keeps the geodesic $\mathbb{H}_{x w}^{1}$ 
only if $\alpha=\beta=0$, which is impossible since $\lambda<0$ by Proposition 16 and $2 \lambda=-\left(\alpha^{2}+\beta^{2}\right)$. So there is no geodesic relative equilibrium. Obviously, the 1-parameter subgroup $B_{\alpha, \beta}(t)$ keeps a 2-dimensional great sphere containing $\mathbb{H}_{x w}^{1}$ only if $\alpha=0$ or $\beta=0$. If $\beta=0$ (resp. $\alpha=0$ ), the associated 2-dimensional relative equilibrium is $B_{ \pm \sqrt{-2 \lambda}, 0}(t) \mathbf{q}$ (resp. $\left.B_{0, \pm \sqrt{-2 \lambda}}(t) \mathbf{q}\right)$.

Let $\mathbf{q}$ be a 2-dimensional ordinary central configuration on $\mathbb{H}_{x y w}^{2}$. Obviously, the 1-parameter subgroup $B_{\alpha, \beta}(t)$ keeps $\mathbb{H}_{x y w}^{2}$ only if $\beta=0$. So 2-dimensional relative equilibria associated with $\mathbf{q}$ is $B_{ \pm \sqrt{-2 \lambda}, 0}(t) \mathbf{q}$. By the discussion before Proposition 21, the proof is complete.

Recall that a relative equilibrium $B_{0, \beta}(t) \mathbf{q}$ is hyperbolic. In [20], Pérez-Chavela and Sánchez-Cerritos consider 2-dimensional hyperbolic relative equilibria. They show that if the masses are equal, the configuration of such relative equilibria could not be a regular polygon. In fact, those motions can be characterized as follows:

Proposition 22. All 2-dimensional hyperbolic relative equilibria must be associated with geodesic ordinary central configurations. Given n masses, there are exactly $n$ ! families of 2-dimensional hyperbolic relative equilibria, one for each ordering of the masses along the geodesic.

Proof. The first part is from the second statement of Proposition 21. We can prove it directly. Since the motion is 2-dimensional, we use the Poincaré half plane model: $H,(x, y), y>0, d s^{2}=\frac{d x^{2}+d y^{2}}{y^{2}}$. Then the kinetic is $K=\frac{1}{2} \sum m_{i} \frac{\dot{x}_{i}^{2}+\dot{y}_{i}^{2}}{y_{i}^{2}}$. In this model, the hyperbolic 1parameter subgroup acts on $H$ by $(x, y) \mapsto e^{\alpha s}(x, y)$ [7]. Thus, the vector filed on $H^{n}$ generated by the hyperbolic 1-parameter subgroup is $\xi_{H^{n}}(\mathbf{q})=\alpha\left(x_{1}, y_{1}, \ldots, x_{n}, y_{n}\right)$. Then the augmented potential is

$$
U+K\left(\xi_{H^{n}}(\mathbf{q})\right)=U+\frac{\alpha^{2}}{2} \sum_{i=1}^{n} m_{i} \frac{x_{i}^{2}}{y_{i}^{2}}+\frac{\alpha^{2}}{2} \sum_{i=1}^{n} m_{i} .
$$

If $\mathbf{q}(t)=e^{\alpha t} \mathbf{q}(0)$ is a hyperbolic relative equilibrium on $H$, then $\mathbf{q}(0)$ is a critical point of the above augmented potential. That is, $\mathbf{q}(0)$ must satisfy the equation

$$
\nabla_{\mathbf{q}_{i}} U=-\frac{\alpha^{2}}{2} \nabla_{\mathbf{q}_{i}} \sum_{i=1}^{n} m_{i} \frac{x_{i}^{2}}{y_{i}^{2}}=-\alpha^{2} m_{i} \frac{1}{y_{i}}\left(x_{i} y_{i},-x_{i}^{2}\right) .
$$

We claim that the critical points of this potential must geodesic configurations. Recall that the geodesics on $H$ are straight lines and circles perpendicular to the $x$-axis. Assume that the particles of $\mathbf{q}(0)$ are distributed on several circular geodesics $x^{2}+y^{2}=R_{j}^{2}, j=1, \ldots, p$ and that 
$R_{j} \leq R_{p}$ if $1 \leq j \leq p$. Consider the equation (11) for one particle, say $\mathbf{q}_{1}$, on largest circle. Note that the right side of (11) is a vector tangent to the largest circle, but the left side, the force exerted on $\mathbf{q}_{1}$, is pointing inwards since there are particles on some smaller circle. This contradiction shows that the critical points of the augmented potential has to be geodesic configurations.

The second part is from Theorem 12 .

Note that for all 2-dimensional hyperbolic relative equilibria, the velocities are orthogonal to the geodesic containing the configuration. This is true for any relative equilibria associated a geodesic configuration. By Proposition 21, we may assume that the geodesic is $\mathbb{H}_{x w}^{1}$. Then the velocity of the $i$-th particle is $\left(0, \alpha x_{i}, \beta w_{i}, 0\right)$ for some $\alpha, \beta$, which is orthogonal to the geodesic.

Proposition 23. For the curved $n$-body problem in $\mathbb{S}^{3}$, consider the relative equilibria associated with ordinary central configurations. Let $\tau$ be the isometry $\tau(x, y, z, w)=(z, w, x, y)$ and $G_{2}=S O(2) \times S O(2)$.

- There is no geodesic relative equilibrium.

- Any 2-dimensional relative equilibria must be in one of the following four forms:

- $g A_{ \pm \sqrt{-2 \lambda}, 0}(t) \mathbf{q}$ and $\tau g A_{ \pm \sqrt{-2 \lambda}, 0}(t) \mathbf{q}$ for $\mathbf{q}$ being a geodesic ordinary central configuration on $\mathbb{S}_{x z}^{1}$ with multiplier $\lambda<0$.

- $g A_{ \pm \sqrt{-2 \lambda}, 0}(t) \mathbf{q}$ and $\tau g A_{ \pm \sqrt{-2 \lambda}, 0}(t) \mathbf{q}$ for $\mathbf{q}$ being a 2-dimensional ordinary central configuration on $\mathbb{S}_{x y z}^{2}$ with multiplier $\lambda<$ 0 .

where $g$ is some isometry in $G_{2}$.

- Any other relative equilibrium is 3-dimensional .

We omit the proof since it is similar to that of Proposition 21. Note that the ordinary central configurations on $\mathbb{S}_{x y z}^{2}$ with multiplier $\lambda>0$ lead to 3-dimensional relative equilibria. Similar to the hyperbolic case, the velocity of any relative equilibrium associated a geodesic configuration is orthogonal to the geodesic containing the configuration.

We now turn to the special central configurations. In this case, the velocity of relative equilibrium associated a geodesic configuration can be along or not along the geodesic containing the configuration. There is no need to discuss the dimension of equilibrium solutions.

Proposition 24. For the curved $n$-body problem in $\mathbb{S}^{3}$, consider the relative equilibria associated with special central configurations.

- Any geodesic relative equilibrium must be $A_{\alpha, 0}(t) \mathbf{q}$, or $\tau A_{\alpha, 0}(t) \mathbf{q}, \alpha \neq$ $\mathbb{R}$ for $\mathbf{q}$ being a special central configuration on $\mathbb{S}_{x y}^{1}$. 
- There is no 2-dimensional relative equilibrium.

- Any other relative equilibrium is 3-dimensional.

Proof. The symmetry group for special central configurations is $O(4)$. Any geodesic special central configuration is in the form of $g \mathbf{q}$, where $\mathbf{q}$ is on $\mathbb{S}_{x y}^{1}$ and $g \in O(4)$. If $g \mathbf{q}$ is on $\mathbb{S}_{x y}^{1}$ (resp. $\mathbb{S}_{z w}^{1}$ ), by Proposition 20, the associated relative equilibria are $A_{\alpha, \beta}(t) \mathbf{q}$ for any $\alpha, \beta \in \mathbb{R}$, which are the same as $A_{\alpha, 0}(t) \mathbf{q}\left(\operatorname{resp} . \tau A_{\alpha, 0}(t) \mathbf{q}\right)$. If $g \mathbf{q}$ is not on $\mathbb{S}_{x y}^{1}$ nor on $\mathbb{S}_{z w}^{1}$, by Proposition 20, the associated relative equilibria are $A_{\alpha, \pm \alpha}(t) \mathbf{q}$, $\alpha \in \mathbb{R}$. The relative equilibrium is geodesic only if $\alpha=0$, and it is not geodesic nor 2-dimensional otherwise.

Let $\mathbf{q}$ be a 2-dimensional special central configuration. We claim that q can not be within $\mathbb{S}_{x y}^{1} \cup \mathbb{S}_{z w}^{1}$. Note that the particles on one of the two circles must be collinear since the configuration is contained in a 3-dimensional hyperplane. Assume that the particles on $\mathbb{S}_{x y}^{1}$ is collinear. The number of particles on $\mathbb{S}_{x y}^{1}$ is one otherwise $\mathbf{q} \in \Delta_{+}$. Then all particles of $\mathbf{q}$ is within one 2-dimensional hemisphere, which is impossible, see Section 12.3 of [6] or [28]. The contradiction proves the claim. Hence, the associated relative equilibria must be $A_{\alpha, \pm \alpha}(t) \mathbf{q}$, $\alpha \in \mathbb{R}$. The relative equilibrium is 2 -dimensional only if $\alpha=0$.

Many researches study the relative equilibria of the curved $n$-body problem by restricting on a 2-dimensional physical space directly, i.e., on $T\left(\left(\mathbb{S}^{2}\right)^{n}-\Delta_{+}\right)$or $T\left(\left(\mathbb{H}^{2}\right)^{n}-\Delta_{-}\right)$. It seems more convenient to start with the configurations, and to use the augmented potentials introduced in Theorem 1 in some proper coordinates. Moreover, this restriction would make the configurations counting clumsy.

Let us finish the discussion with one concrete example. In [8], Diacu and Sergiu consider 3-dimensional relative equilibria of three-body in $\mathbb{S}^{3}$ with the property: The configuration is not geodesic and the three mutual distances are the same. They show that the three masses must be equal. This result can be obtained quickly as follows:

The associated central configuration must be 2-dimensional since there are only three bodies and the configuration is not geodesic. It can not be a special central configuration since it is not geodesic [28]. We may assume that it is on $\mathbb{S}_{x y z}^{2}$, i.e., $\mathbf{q}_{i}=\left(x_{i}, y_{i}, z_{i}\right)$. By Diacu and Zhu [10], a three-body configuration on $\mathbb{S}_{x y z}^{2}$ is an ordinary central configuration if and only if

$\sum m_{i} z_{i} x_{i}=\sum m_{i} z_{i} y_{i}=0, \quad\left(z_{1}, z_{2}, z_{3}\right)=k\left(\sin ^{3} d_{23}, \sin ^{3} d_{13}, \sin ^{3} d_{12}\right)$.

Since the three mutual distances are the same, we get immediately that the three masses are the same. 


\section{ACKNOWLEDGMENT}

I would like to thank Xiang Yu and Ernesto Pérez-Chavela for careful reading of the manuscript and helpful suggestions. I would like to thank the referee for many helpful suggestions and pointing out a gap in the original manuscript. This work is supported by NSFC(No.11801537) and China Scholarship Council (CSC NO. 201806345013).

\section{REFERENCES}

[1] A. Albouy, V. Kaloshin, Finiteness of central configurations of five bodies in the plane, Annals of Mathematics 176 (2012), no. 1, 535-588.

[2] V.I. Arnold, V.V. Kozlov, A.I. Neishtadt, Mathematical Aspects of Classical and Celestial Mechanics. [Dynamical Systems. III], Translated from the Russian original by E. Khukhro, 3rd edition, Encyclopaedia of Mathematical Sciences, 3. Springer-Verlag, Berlin, 2006.

[3] A.V. Borisov, I.S. Mamaev, A.A. Kilin, Two-body problem on a sphere. reduction, stochasticity, periodic orbits, Regul. Chaotic Dyn. 9 (2004), no. 3, 265-279.

[4] A.V. Borisov, L.C. García-Naranjo, I.S. Mamaev, J. Montaldi, Reduction and relative equilibria for the two-body problem on spaces of constant curvature. Celestial Mech. Dynam. Astronom. 130 (2018), no. 6, 1-36.

[5] A. Chenciner, The angular momentum of an ordinary central configuration, Discrete Contin. Dyn. Syst. 33 (2013), no. 3, 1033-1047.

[6] F. Diacu, Relative equilibria of the 3 -dimensional curved $n$-body problem, Memoirs Amer. Math. Soc. 228 (2013), no. 1071.

[7] F. Diacu, E. Pérez-Chavela, J. Guadalupe Reyes Victoria, An intrinsic approach in the curved $n$-pody problem. the negative curvature case, J. Differential Equations 252 (2012), no. 8, 4529-4562.

[8] F. Diacu, P. Sergiu, All the Lagrangian relative equilibria of the curved 3-body problem have equal masses, J. Math. Phys. 55 (2014), no. 11.

[9] F. Diacu, C. Stoica, S. Zhu, Central configurations of the curved $n$-body problem, J. Nonlinear Sci., 28 (2018), no. 5, 1999-2046.

[10] F. Diacu, S. Zhu, Almost all 3-body relative equilibria are inclined, Discrete Contin. Dyn. Syst. Ser. S. 13 (2020), no. 4, 1131-1143.

[11] L.C. García-Naranjo, J.C. Marrero, E. Pérez-Chavela, M. Rodríguez-Olmos, Classification and stability of relative equilibria for the two-body problem in the hyperbolic space of dimension 2, J. Differential Equations 260 (2016), no. 7, 6375-6404.

[12] A.A. Kilin, Libration points in spaces $\mathbf{S}^{2}$ and $\mathbf{L}^{2}$, Regul. Chaotic Dyn. 4 (1999), no. 1, 91-103.

[13] R. Martínez, C. Simó, Relative equilibria of the restricted three-body problem in curved spaces, Celestial Mech. Dynam. Astronom. 128(2017), no. 2-3, 221259.

[14] R. Moeckel, Celestial Mechanics-Especially Central Configurations, unpublished lecture notes.

http://www.math.umn.edu/ ${ }^{\sim}$ rmoeckel/notes/CMNotes.pdf 
[15] J. Marsden, Lectures on Mechanics. London Mathematical Society Lecture Note Series, 174. Cambridge University Press, Cambridge, 1992.

[16] F.R. Moulton, The straight line solutions of $n$ bodies, Ann. of Math. (2) 12 (1910), no. 1, 1-17.

[17] F. Pacella, Central configurations of the $n$-body problem via equivariant Morse theory, Arch. Rational Mech. Anal. 97 (1987), no. 1, 59-74.

[18] J.I. Palmore, Classifying relative equilibria. I, Bull. AMS 79(1973), 904-908.

[19] J.I. Palmore, Relative equilibria of the n-body problem in $E^{4}$. J. Differential Equations 38 (1980), no. 2, 278-300.

[20] E. Pérez-Chavela, J.M. Sánchez-Cerritos, Hyperbolic relative equilibria for the negative curved $n$ body problem, Commun. Nonlinear Sci. Numer. Simul. 67 (2019), 460-479.

[21] M. Shub, Diagonals and relative equilibria, Appendix to Smale's paper, in Manifolds-Amsterdam, Springer Lecture Notes in Math. 197(1970), 199-201.

[22] S. Smale, Topology and mechanics, II. The planar $n$-body problem, Invent. Math. 11 (1970), 4564.

[23] S. Smale, Problems on the nature of relative equilibria in celestial mechanics, in Manifolds-Amsterdam, Springer Lecture Notes in Math. 197(1970), 194-198.

[24] P. Tibboel, Existence of a lower bound for the distance between point masses of relative equilibria in spaces of constant curvature, J. Math. Anal. Appl. 416 (2014), no. 1, 205-211.

[25] A. Wintner, The Analytical Foundations of Celestial Mechanics, Princeton University Press, Princeton, N.J., 1947.

[26] X. Yu, S. Zhu, Regular polygonal equilibrium configurations on $\mathbb{S}^{1}$ and stability of the associated relative equilibria, J. Dynam. Differential Equations (2020), https://doi.org/10.1007/s10884-020-09848-1

[27] S. Zhu, S. Zhao, 3-dimensional central configurations in $\mathbb{H}^{3}$ and $\mathbb{S}^{3}$, J. Math. Phys. 58 (2017), no. 2, 022901.

[28] S. Zhu, Dziobek equilibrium configurations on a sphere, arXiv:1705.03987. 